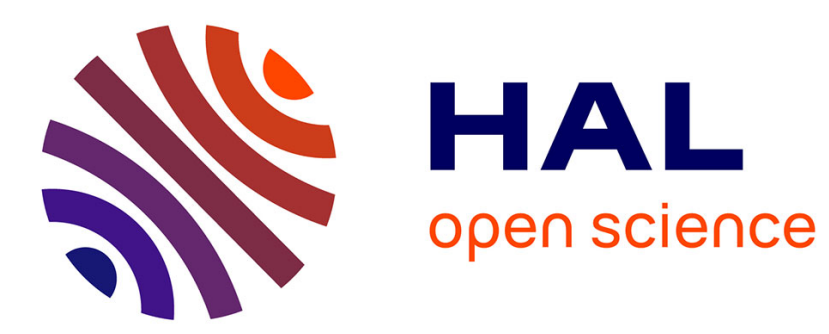

\title{
Analytical and numerical approaches to nonlinear galloping of internally-resonant suspended cables
}

Angelo Luongo, Daniele Zulli, Giuseppe Piccardo

\section{To cite this version:}

Angelo Luongo, Daniele Zulli, Giuseppe Piccardo. Analytical and numerical approaches to nonlinear galloping of internally-resonant suspended cables. Journal of Sound and Vibration, 2008, 315 (3), pp.375-393. hal-00788555

\section{HAL Id: hal-00788555 \\ https://hal.science/hal-00788555}

Submitted on 14 Feb 2013

HAL is a multi-disciplinary open access archive for the deposit and dissemination of scientific research documents, whether they are published or not. The documents may come from teaching and research institutions in France or abroad, or from public or private research centers.
L'archive ouverte pluridisciplinaire HAL, est destinée au dépôt et à la diffusion de documents scientifiques de niveau recherche, publiés ou non, émanant des établissements d'enseignement et de recherche français ou étrangers, des laboratoires publics ou privés. 


\title{
Analytical and numerical approaches to nonlinear galloping of internally resonant suspended cables
}

\author{
Angelo Luongo ${ }^{a}$, Daniele Zulli,**, Giuseppe Piccardo ${ }^{\mathrm{b}}$ \\ ${ }^{\mathrm{a} D I S A T}$ University of L'Aquila, 67040 L'Aquila, Italy \\ ${ }^{\mathrm{b}}$ DICAT University of Genoa, 16145 Genova, Italy
}

\begin{abstract}
A study is carried out on nonlinear multimodal galloping of suspended cables. A consistent model of a curved cable beam, geometrically nonlinear and able to torque, recently formulated by the authors, is used. The model accounts for quasi steady aerodynamic forces, including the effect of static swing of the cable and dynamic twist of the cross section. Complementary solution methods are employed, namely, finite difference and Galerkin spatial discretization, followed by numerical time integration, or Galerkin spatial discretization in conjunction with Multiple Scale perturbation analysis. The different techniques are applied to a cable close to the first cross over point, at which a number of internal resonances exist. Branches of periodic solutions and their stability are evaluated as functions of wind velocity. The existence of branches of quasi periodic solutions, originating from narrow unstable intervals and propagating elsewhere, is also proved. Qualitative and quantitative results furnished by the different investigation tools are compared among them, and the importance of the various components of motion, accounted or neglected in the reduced models, is discussed.
\end{abstract}

(C) 2008 Elsevier Ltd. All rights reserved.

\section{Introduction}

The aerodynamic instability in the quasi-steady regime (called galloping in technical language) is a classic phenomenon of aerodynamic instability of slender structures having non-circular cross-sections. Typical structural systems susceptible to galloping are suspended cables subjected to icing conditions, which suffer instability even at a moderate wind velocity. Galloping manifests itself by oscillations of great amplitude that may become stable on limit cycles or divergent over time, according to the nonlinear characteristics of the system.

In the technical literature the phenomenon is usually described by one-degree-of-freedom models (mono-modal galloping), which can be derived from a sectional model (e.g. Ref. [1]) or deduced by discretizing a continuous model (e.g. Ref. [2]). In these cases one assumes that the ignored modes are stable and provide a marginal contribution to the motion. However, when the natural frequencies of the mechanical

\footnotetext{
*Corresponding author.

Email addresses: luongo@ing.univaq.it (A. Luongo), danzulli@,ing.univaq.it (D. Zulli), giuseppe.piccardo@unige.it (G. Piccardo).
} 
model are in internal resonance conditions $(1: 1,1: 2$, etc.), more modes, stable or unstable, can be involved in the motion, thus giving rise to multimodal galloping conditions.

Concerning aerodynamics, in the framework of the quasi-steady theory, it is well known that forces exerted by a fluid on non-circular rigid cylinders depend on the body exposure with respect to the fluid direction. When elastic beams are considered, it is usual to calculate the aerodynamic forces by referring to the initial attitude of the section; the instantaneous rotation is taken into account only to determine, in an approximate way, the fluid-to-structure relative velocity. This approach is used for models of perfectly flexible strings too, in which rotations are generally considered not relevant to the structural behavior. On the contrary, for sagged suspended cables, the problem appears more complicated. Because of the great structural flexibility, the steady component of aerodynamic forces leads to significant variations in the equilibrium configuration of suspended cables and, then, in their flow attitude. Therefore, besides the dynamic rotation, a static swing has to be considered as a function of the mean wind velocity.

The aeroelastic instability of suspended cables has been widely discussed in the literature, with rare attempts of including a twist angle in the treatment, in any case neglecting the intrinsic coupling with the flexure. Recently, the authors have formulated a consistent linear model of cable-beam [3], which takes into account the initial curvature together with flexural and torsional stiffness, and both static and dynamic torsion. Afterwards, they have reformulated the model in the nonlinear field [4,5], obtaining equations identical (at the main order) to those of a flexible suspended cable (e.g. Ref. [6]), but with the addition of a fourth equation, that can be interpreted as the rotational equilibrium in the tangential direction of a planar circular arch (e.g. Ref. [7]).

In the present paper, use is made of the nonlinear model of cable-beam proposed by the authors, to investigate the nonlinear galloping behavior of a suspended cable, under internal resonance conditions, devoting attention to the comparison between analytical and numerical approaches. A cable close to the first cross-over point is considered, for which the first symmetric in-plane mode is in internal resonance of 1:1 type with the first anti-symmetric in-plane and out-of-plane modes, and of 2:1 type with the first symmetric out-ofplane mode [8]. The problem is tackled in three different ways. First, (a) the nonlinear integro-partialdifferential equations are spatially discretized by the finite-difference method and numerically integrated in time. Then a standard Galerkin method is applied to obtain a few-dof discrete model, whose solutions are drawn both (b) numerically, through direct integrations of equations, and (c) analytically, by using the Multiple-Scale perturbation Method. An indepth analysis is performed on a case study, for which analytical and numerical solutions are compared. Finally, the stability of nonlinear branches is investigated, and the importance of analytical solutions in an understanding of the phenomenon is highlighted.

\section{A nonlinear model of curved cable-beam}

The cable is modeled as a body made of a flexible centerline and rigid cross-sections, restrained to remain orthogonal to the axis (shear-undeformable beam). An ice accretion is considered, assumed to be uniform along the cable. Under the gravity force $\mathbf{b}_{0}$, which includes own and ice weights, the cable hangs in the vertical plane and takes on the $\mathscr{C}_{0}$ configuration (Fig. 1a). A wind flow is then considered, of mean velocity $\mathbf{U}=U \mathbf{a}_{z}$, blowing normal to the cable plane, producing aerodynamic forces which can be (approximately) taken to be uniform along the centerline, if lightly sagged cables are considered. When the static part of these forces is vectorially summed with the gravity, a uniform force field $\mathbf{b}$ is obtained, parallel to an inclined plane. The force induces a rotation $\varphi(U)$ of the cable as a rigid body, referred in the following as the static swing of the cable (Fig. 1b). The inclined planar configuration is taken as a reference $\mathscr{C}$ configuration, and dynamic displacements are measured from it. When the dynamic part of the aerodynamic forces is considered, due to the occurrence of the inertia forces, the cable loses its planar configuration.

The incremental equilibrium equations around the reference pre-stressed state have been derived in Refs. [4,5]. By using a linear elastic constitutive law and accounting for damping and inertial effects, the complete equations of motion have been obtained. They have successively been strongly simplified by estimating the order of magnitude of all their terms, under the hypotheses of a small sag-to-span ratio, high slenderness, compact section and small transversal-to-longitudinal and transversal-to-torsional wave velocity ratios. The latter assumption has permitted to neglect tangential and torsional forces and couples, both of inertial and 
(a)

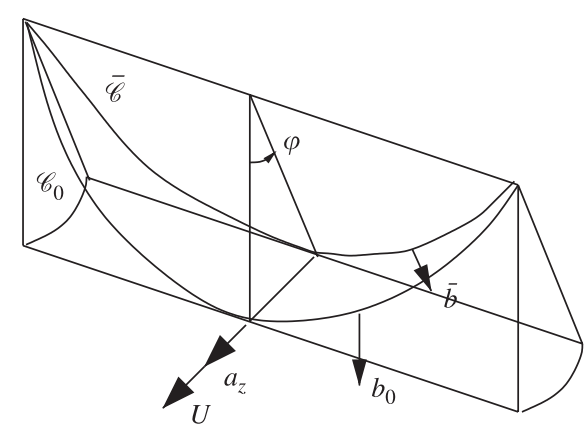

(b)

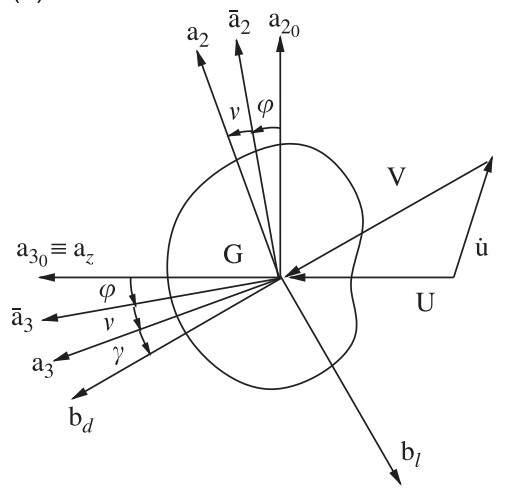

Fig. 1. (a) Initial $\mathscr{C}_{0}$ and reference $\overline{\mathscr{C}}$ configurations $\left(\mathbf{b}_{0}=\right.$ self weight; $\overline{\mathbf{b}}=$ s.w. + wind steady forces); (b) aerodynamic forces on the transversal section $\left(\mathbf{V}=\mathbf{U} \quad \dot{\mathbf{u}}=\right.$ relative wind velocity; $\gamma=$ angle of attack; $\mathbf{b}_{d}, \mathbf{b}_{l}=$ drag and lift forces).

damping nature. A nonlinear curved cable-beam model has thus been deduced:

$$
\begin{aligned}
& -\frac{E A}{\ell}\left(\kappa+v^{\prime \prime}\right) \int_{0}^{\ell}\left(\kappa v-\frac{1}{2} v^{\prime 2}-\frac{1}{2} w^{\prime 2}\right) \mathrm{d} s+T v^{\prime \prime}+b_{2}-m \ddot{v}-c_{v} \dot{v}=0 \\
& -\frac{E A}{\ell} w^{\prime \prime} \int_{0}^{\ell}\left(\kappa v-\frac{1}{2} v^{\prime 2}-\frac{1}{2} w^{\prime 2}\right) \mathrm{d} s+T w^{\prime \prime}+b_{3}-m \ddot{w}-c_{w} \dot{w}=0 \\
& G J \vartheta^{\prime \prime}-E I \kappa^{2} \vartheta+(E I+G J) \kappa w^{\prime \prime}-E I \kappa \vartheta v^{\prime \prime}+G J\left(v^{\prime \prime} w^{\prime}\right)^{\prime}=0 \\
& v=0, \quad w=0, \quad G J\left(\vartheta^{\prime}+\kappa w^{\prime}+w^{\prime} v^{\prime \prime}\right)=0 \quad \text { at } s=0, \ell
\end{aligned}
$$

where the integrals arise from the static condensation of the tangential displacements. In Eqs. (1) $v(s, t), w(s, t)$ are the in-plane and out-of-plane translational displacements measured from the reference rotated configuration $\mathscr{C} ; \vartheta(s, t)$ is the dynamic twist angle; $T$ and $\kappa$ are the stress and curvature in the reference configuration, assumed to be constant along the cable; $\ell$ and $m$ are the cable length and mass per-unitlength; $c_{v}$ and $c_{w}$ are the structural damping coefficients; $E A, G J$ and $E I$ are the axial, torsional and flexural stiffnesses; the dots and dashes denote differentiation with respect to the time $t$ and the curvilinear abscissa $s$, respectively; and $b_{2}$ and $b_{3}$ are aerodynamic, time-dependent, external forces. The latter have been evaluated according to a quasi-steady formulation (Fig. 1b). A simplified model has been adopted by neglecting the cable curvature, the flexural rotations and the aerodynamic couples, which would cause rotations $\vartheta$ much smaller than the static swing $\varphi$. Moreover, the relative wind velocity $\mathbf{V}$ (Fig. 1b) has been evaluated ignoring the twist velocity $\dot{\vartheta}$, which entails velocities at the cross-section boundary smaller than the centerline velocities $\dot{w}$ and $\dot{v}$ [3]. In conclusion, the aerodynamic force $\mathbf{b}_{a}=\mathbf{b}_{d}+\mathbf{b}_{l}$ is a function of the mean wind velocity $U$, of the twist, of the translational velocities, and of the static swing, which in turn depends on $U$; namely $\mathbf{b}_{a}=\mathbf{b}_{a}(\vartheta, \dot{v}, \dot{w} ; \varphi(U), U)$. The force components $b_{2}$ and $b_{3}$ are derived by projecting the force $\mathbf{b}_{a}$ on the reference axes, $\mathbf{a}_{2}$ and $\mathbf{a}_{3}$, respectively, and expanding for small $\vartheta, \dot{v}$ and $\dot{w}$, up to cubic terms. Moreover, the steady part of $\mathbf{b}_{a}$ is considered to evaluate, through equilibrium conditions, the static swing $\varphi$ and the stress $T$ (see Appendix A).

It is worth noticing that, when Eqs. (1) are linearized and damping and aerodynamic forces are neglected (linear Hamiltonian system), two classes of motion are found, namely in-plane (no torsion) modes and out-ofplane plus torsion modes. Therefore, no purely torsional modes exist and torsion is only coupled with out-ofplane displacements. This is due to the small (but finite) curvature of the cable. Indeed, when a twist moment arises, an out-of-plane bending moment is triggered for equilibrium reasons, as it appears from Eq. (1) $)_{3}$. In contrast, an in-plane bending moment is equilibrated by shear forces only, but both these effects are of a higher order with respect to the prestress, so that they do not appear in Eqs. (1) 1,2 . Of course, when nonlinearities are accounted for, in-plane and out-of-plane (plus torsion) modes also couple among them. 


\section{Analytical and numerical approaches}

The integro-differential equations (1) are solved here by both numerical and analytical approaches. Different types of analyses are performed, by using the finite-difference and the Galerkin methods, the latter in conjunction with a numerical integration or a perturbation (Multiple Scale) solution. The aim of the analysis is to investigate, on the one hand, the ability of a suitably selected small-dimensional system to capture the essential dynamics of the large-dimensional system and, on the other, the efficiency of an approximate analytical approach in describing the complex bifurcation scenario, when compared with a purely numerical approach.

\subsection{Finite-difference discretization}

The finite-difference method is applied to perform a spatial discretization of Eqs. (1). Constant-spacing $(n+1)$ nodes are considered, at the abscissas $s_{i}=i \Delta(i=0,1, \ldots, n)$, with $\Delta:=\ell / n$ the length of the subintervals (Fig. 2).

The translational displacements $v_{i}(t):=v\left(s_{i}, t\right)$ and $w_{i}(t):=w\left(s_{i}, t\right)$ and the twist angles $\vartheta_{i}(t):=\vartheta\left(s_{i}, t\right)$ at nodes, functions of the sole time $t$, are taken as the unknowns of the problem. The central differences are used to discretize the spatial derivatives inside the domain $(i=1,2, \ldots, n-1)$, whereas the forward and backward differences are employed to express the mechanical boundary conditions $(1)_{6}$ at $i=0, n$. The integrals appearing in Eqs. (1) are approximated by the Newton-Cotes formula:

$$
\int_{0}^{\ell}() \mathrm{d} s=\Delta\left[\frac{17}{18}()_{0}+\frac{59}{18}()_{1}+\frac{43}{18}()_{2}+\frac{49}{18}()_{3}+\sum_{i=4}^{n}()_{i}+\cdots\right]
$$

The whole procedure, able to tackle an arbitrary number of nodes over the cable length, has been developed through the symbolic software Mathematica ${ }^{\circledR}$ [9]. The resultant $3(n+1)$ equations of mixed algebraicdifferential type $(3(n-1)$ differential and 6 algebraic) are numerically integrated in time by a procedure fitted for such kinds of systems [10]. In particular, the motion of two selected nodes $(s=\ell / 4$ and $s=\ell / 2)$ is recorded in order to compare it with the results of the other methods illustrated in the following.

The Rayleigh formula is used to introduce the structural damping (see e.g. Ref. [11]). To this end, the continuous damping coefficients $c_{v}$ and $c_{w}$ in Eqs. (1) are ignored and damping is directly introduced into the discrete model as $\mathbf{C}_{s}:=\alpha \mathbf{M}+\beta \mathbf{K}$, with $\mathbf{M}$ and $\mathbf{K}$ the mass and the stiffness matrix, respectively. Since the mass is non-zero only at the internal nodes $i=1,2, \ldots, n-1$, stiffness is also considered only at these nodes.

\subsection{Galerkin discretization}

An alternative (generally low-dimensional) discrete model is derived from Eqs. (1) by using a standard Galerkin procedure, namely:

$$
\left(\begin{array}{c}
\vartheta(s, t) \\
v(s, t) \\
w(s, t)
\end{array}\right)=\sum_{j=1}^{m}\left(\begin{array}{c}
0 \\
\phi_{v_{j}} \\
0
\end{array}\right) q_{j}^{i}(t)+\sum_{k=1}^{n}\left(\begin{array}{c}
\phi_{\vartheta_{k}} \\
0 \\
\phi_{w_{k}}
\end{array}\right) q_{k}^{o}(t) .
$$

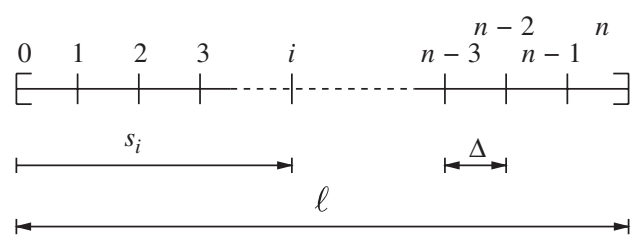

Fig. 2. Spatial discretization in the finite difference method. 
Here, the in-plane $\phi_{v_{j}}$ and out-of-plane $\left(\phi_{\vartheta_{k}}, \phi_{w_{k}}\right)$ eigenfunctions, deduced from the associated linearized Hamiltonian problem, are taken as trial functions. Moreover, $q_{j}^{i}(t),(j=1, \ldots, m)$, and $q_{k}^{o}(t),(k=1, \ldots, n)$ are the unknown amplitudes for the in-plane and out-of-plane modes, respectively. By replacing Eqs. (3) in the weak form of Eqs. (1), the following nonlinear discretized model is obtained:

$$
\begin{aligned}
\ddot{\mathbf{q}}+\mathbf{K q}= & -\mathbf{C} \dot{\mathbf{q}}-\mathbf{H q}+\mathbf{N}_{0}[\mathbf{q}, \mathbf{q}]+\mathbf{N}_{1}[\mathbf{q}, \dot{\mathbf{q}}]+\mathbf{N}_{2}[\dot{\mathbf{q}}, \dot{\mathbf{q}}] \\
& +\mathbf{M}_{0}[\mathbf{q}, \mathbf{q}, \mathbf{q}]+\mathbf{M}_{1}[\mathbf{q}, \mathbf{q}, \dot{\mathbf{q}}]+\mathbf{M}_{2}[\mathbf{q}, \dot{\mathbf{q}}, \dot{\mathbf{q}}]+\mathbf{M}_{3}[\dot{\mathbf{q}}, \dot{\mathbf{q}}, \dot{\mathbf{q}}]
\end{aligned}
$$

where $\mathbf{q}=\left(q_{j}^{i}(t), q_{k}^{o}(t)\right)$ is the $(m+n)$-vector of the Lagrangian parameters; $\mathbf{K}, \mathbf{C}$ and $\mathbf{H}$ are the stiffness, damping (structural plus aerodynamic) and circulatory matrices, respectively, which are of block-diagonal type, because of the symmetric-anti-symmetric character of cable eigenfunctions; and $\mathbf{N}_{p}(p=0,1,2)$ and $\mathbf{M}_{r}(r=0,1,2,3)$ are bilinear and trilinear operators collecting quadratic and cubic nonlinearities, respectively, both of mechanical and aerodynamic types. The explicit expressions for the coefficients of Eq. (4), relevant to a strongly reduced 2 dof system $(m=2, n=0)$, used in the following, are given in Appendix B. Larger dimensional systems, also employed in the following, are not reported for the sake of brevity.

A solution to Eqs. (4) has first been obtained through direct numerical integration, by using standard routines [10]. Stable motions are roughly estimated by starting the integration procedure with generic initial conditions, and checking the steady-motion amplitude when the transient response is exhausted.

\subsection{Multiple Scale method}

The Multiple Scale perturbation Method (MSM, see e.g. Ref. [12]) has been applied to the discretized equations (4). As it is well known, the method furnishes equations that govern the slow flow of the system, filtering its fast dynamics. As a result, analytical solutions are possibly drawn, their stability is more easily studied and numerical integrations are more easily performed.

A dimensionless perturbation parameter $\varepsilon \ll 1$ is introduced, and the vector $\mathbf{q}$ is expanded in series as $\mathbf{q}=\varepsilon \mathbf{q}_{1}+\varepsilon^{2} \mathbf{q}_{2}+\varepsilon^{3} \mathbf{q}_{3}+\cdots$. Moreover, it is assumed that the coefficients of the matrices $\mathbf{C}$ and $\mathbf{H}$ are small of the same order $\varepsilon$, namely $\mathbf{C}=\varepsilon \tilde{\mathbf{C}}$ and $\mathbf{H}=\varepsilon \tilde{\mathbf{H}}$, with $\mathcal{O}(\|\tilde{\mathbf{C}}\|)=\mathcal{O}(\|\tilde{\mathbf{H}}\|)=1$. Two independent slow time scales, $t_{1}=\varepsilon t$ and $t_{2}=\varepsilon^{2} t$, are introduced in addition to the fast scale $t_{0}=t$, so that, by the chain rule, $\mathrm{d} / \mathrm{d} t=$ $d_{0}+\varepsilon d_{1}+\varepsilon^{2} d_{2}+\cdots$, with $d_{k}:=\partial / \partial t_{k}$. The perturbation equations, collecting terms with the same power of $\varepsilon$, are of the following type:

$$
\begin{aligned}
\varepsilon: \quad d_{0}^{2} \mathbf{q}_{1}+\mathbf{K} \mathbf{q}_{1}= & \mathbf{0} \\
\varepsilon^{2}: \quad d_{0}^{2} \mathbf{q}_{2}+\mathbf{K} \mathbf{q}_{2}= & -2 d_{0} d_{1} \mathbf{q}_{1}-\tilde{\mathbf{C}} d_{0} \mathbf{q}_{1}-\tilde{\mathbf{H}} \mathbf{q}_{1}+\mathbf{N}_{0}\left[\mathbf{q}_{1}, \mathbf{q}_{1}\right] \\
& +\mathbf{N}_{1}\left[\mathbf{q}_{1}, d_{0} \mathbf{q}_{1}\right]+\mathbf{N}_{2}\left[d_{0} \mathbf{q}_{1}, d_{0} \mathbf{q}_{1}\right] \\
\varepsilon^{3}: \quad d_{0}^{2} \mathbf{q}_{3}+\mathbf{K} \mathbf{q}_{3}= & -2 d_{0} d_{1} \mathbf{q}_{2}-d_{1}^{2} \mathbf{q}_{1}-2 d_{0} d_{2} \mathbf{q}_{1} \\
& -\tilde{\mathbf{C}}\left(d_{0} \mathbf{q}_{2}+d_{1} \mathbf{q}_{1}\right)-\tilde{\mathbf{H}} \mathbf{q}_{2}+2 \mathbf{N}_{0}\left[\mathbf{q}_{1}, \mathbf{q}_{2}\right] \\
& +\mathbf{N}_{1}\left[\mathbf{q}_{1}, d_{0} \mathbf{q}_{2}\right]+\mathbf{N}_{1}\left[\mathbf{q}_{1}, d_{1} \mathbf{q}_{1}\right]+\mathbf{N}_{1}\left[\mathbf{q}_{2}, d_{0} \mathbf{q}_{1}\right] \\
& +\mathbf{N}_{2}\left[d_{0} \mathbf{q}_{1}, d_{0} \mathbf{q}_{2}\right]+\mathbf{N}_{2}\left[d_{0} \mathbf{q}_{2}, d_{0} \mathbf{q}_{1}\right] \\
& +\mathbf{N}_{2}\left[d_{0} \mathbf{q}_{1}, d_{1} \mathbf{q}_{1}\right]+\mathbf{N}_{2}\left[d_{1} \mathbf{q}_{1}, d_{0} \mathbf{q}_{1}\right] \\
& +\mathbf{M}_{0}\left[\mathbf{q}_{1}, \mathbf{q}_{1}, \mathbf{q}_{1}\right]+\mathbf{M}_{1}\left[\mathbf{q}_{1}, \mathbf{q}_{1}, d_{0} \mathbf{q}_{1}\right] \\
& +\mathbf{M}_{2}\left[\mathbf{q}_{1}, d_{0} \mathbf{q}_{1}, d_{0} \mathbf{q}_{1}\right]+\mathbf{M}_{3}\left[d_{0} \mathbf{q}_{1}, d_{0} \mathbf{q}_{1}, d_{0} \mathbf{q}_{1}\right]
\end{aligned}
$$

Eq. (5) $)_{1}$ admits the solution:

$$
\mathbf{q}_{1}=\sum_{l=1}^{m+n} A_{l}\left(t_{1}, t_{2}\right) \mathbf{u}_{l} \mathrm{e}^{\mathrm{i} \omega_{l} t}+\text { c.c. }
$$

where $i$ is the imaginary unit, $A_{l}$ are unknown complex amplitudes depending on the slow time scales, $\mathbf{u}_{l}$ are the eigenvectors, $\omega_{l}$ the associated frequencies and c.c. denotes the complex conjugate. By substituting Eq. (6) 
into Eq. $(5)_{2}$, a number of (resonant) terms that would lead to divergent solutions on the $t_{0}$-scale (secular terms) arise. In selecting them, all the internal resonances among the frequencies $\omega_{l}$ must be taken into account. By zeroing the secular terms, a set of differential equations of the type:

$$
d_{1} A_{l}=\mathscr{F}_{1 l}\left(A_{1}, A_{2}, \ldots, A_{m+n}\right) \quad l=1,2, \ldots, m+n
$$

follow. Then, by solving for $\mathbf{q}_{2}$ and removing secular terms at the higher order, conditions of similar type are found:

$$
d_{2} A_{l}=\mathscr{F}_{2 l}\left(A_{1}, A_{2}, \ldots, A_{m+n}\right) \quad l=1,2, \ldots, m+n
$$

Hence, by recombining Eqs. (7) and (8) to come back to the true time $t$, and re-absorbing the parameter $\varepsilon$, it follows:

$$
\dot{A}_{l}=\mathscr{F}_{l}\left(A_{1}, A_{2}, \ldots, A_{m+n}\right) \quad l=1,2, \ldots, m+n
$$

with $\mathscr{F}_{l}=\mathscr{F}_{1 l}+\mathscr{F}_{2 l}$. Eqs. (9) are called Amplitude Modulation Equations (AME) and govern the slow dynamics of the system.

As an example, which will be extensively used in the following, when a discrete system (4) with only two dof's is considered $(m+n=2)$, having Hamiltonian frequencies $\omega_{1}$ and $\omega_{2}$ in 1:1 internal resonance (namely, $\omega_{1}=\omega_{2}+\varepsilon \sigma$, with $\sigma=\mathcal{O}(1)$ a detuning parameter), the AME (9) turn out to be

$$
\begin{aligned}
\dot{A}_{1}= & A_{1}\left[-\frac{1}{2}\left(c_{a 1}+2 \xi_{1} \omega_{1}\right)+\mathrm{i} p_{1}\right]+A_{1} A_{2}^{2}\left(p_{21}+\mathrm{i} p_{22}\right) \mathrm{e}^{2 \mathrm{i} \sigma t} \\
& +A_{1} A_{2} A_{2}\left(p_{31}+\mathrm{i} p 32\right)+A_{1}^{2} A_{1}\left(p_{41}+\mathrm{i} p_{42}\right) \\
\dot{A}_{2}= & A_{2}\left[-\frac{1}{2}\left(c_{a 2}+2 \xi_{2} \omega_{2}\right)+\mathrm{i} p_{6}\right]+A_{1}^{2} A_{2}\left(p_{71}+\mathrm{i} p_{72}\right) \mathrm{e}^{2 \mathrm{i} \sigma t} \\
& +A_{1} A_{1} A_{2}\left(p_{61}+\mathrm{i} p_{62}\right)+A_{2}^{2} A_{2}\left(p_{81}+\mathrm{i} p_{82}\right)
\end{aligned}
$$

where $A_{1}$ and $A_{2}$ are the complex amplitudes of the symmetric and anti-symmetric in plane modes, respectively, and the coefficients $p_{i}=p_{i}(U)$ are given in Appendix $\mathrm{C}$.

The AME can be put in real form by adopting polar, cartesian or mixed forms for the complex amplitudes (see Ref. [13] for a general discussion on algorithmic problems arising with such forms). If the polar representation is adopted, namely $A_{l}=\frac{1}{2} a_{l} \exp \left(\mathrm{i} \alpha_{l}\right)$, with $a_{l}$ the amplitudes and $\alpha_{l}$ the phases, the introduction of phase differences permits to further reduce the system dimension, and to transform it into an autonomous form. For example, when the procedure is applied to Eqs. (10), by letting $\psi:=2\left(\alpha_{1}-\alpha_{2}-\sigma t\right)$, the following Reduced Amplitude Modulation Equations (RAME) are obtained:

$$
\begin{aligned}
\dot{a}_{1}= & -\frac{1}{2} a_{1}\left(c_{a 1}+2 \xi_{1} \omega_{1}\right)+\frac{1}{4} a_{1} a_{2}^{2}\left(p_{21} \cos \psi-p_{22} \sin \psi+p_{31}\right)+\frac{1}{4} a_{1}^{3} p_{41} \\
\dot{a}_{2}= & -\frac{1}{2} a_{2}\left(c_{a 2}+2 \xi_{2} \omega_{2}\right)+\frac{1}{4} a_{1}^{2} a_{2}\left(p_{71} \cos \psi+p_{72} \sin \psi+p_{61}\right)+\frac{1}{4} a_{2}^{3} p_{81} \\
a_{1} a_{2} \dot{\psi}= & a_{1} a_{2}\left(2 p_{5}-2 p_{1}-2 \sigma\right)+\frac{1}{2} a_{1}^{3} a_{2}\left(p_{62}-p_{71} \sin \psi+p_{72} \cos \psi-p_{42}\right) \\
& -\frac{1}{2} a_{1} a_{2}^{3}\left(p_{32}+p_{21} \sin \psi+p_{22} \cos \psi-p_{82}\right)
\end{aligned}
$$

The polar form, however, becomes singular when at least one amplitude $a_{l}$ vanishes (see, for instance, Eq. $\left.(11)_{3}\right)$. Therefore, the equations cannot be put in the standard form $\dot{\mathbf{x}}=\mathbf{f}(\mathbf{x})$. This drawback entails that the stability of solutions containing some zero amplitudes cannot be studied via the standard Jacobian eigenvalue analysis, nor can software packages suited for standard form equations be used. To overcome such difficulties, a mixed polar-cartesian form can be adopted [13]. When this procedure is applied to Eqs. (10), by letting $A_{1}=\frac{1}{2}\left(u_{1}+\mathrm{i} v_{1}\right) \exp \left(\mathrm{i} v_{1}\right)$ and $A_{2}=\frac{1}{2} a_{2} \exp \left(\mathrm{i} v_{2}\right)$, with $v_{1}=\alpha_{2}-\sigma t$ and $v_{2}=\alpha_{2}$, and performing some algebraic manipulations, a standard form set of equations in the $\mathbf{x}:=\left(u_{1}, v_{1}, a_{2}\right)$ variables, suitable for the study 
of the stability of solutions with $a_{1}=0$, is obtained:

$$
\begin{aligned}
\dot{u}_{1}= & u_{1}\left[-\frac{1}{2}\left(c_{a 1}+2 \xi_{1} \omega_{1}\right)+\frac{1}{4}\left(p_{21}+p_{31}\right) a_{2}^{2}\right] \\
& +v_{1}\left[p_{5}-p_{1}-\sigma+\frac{1}{4}\left(p_{22}-p_{32}+p_{82}\right) a_{2}^{2}\right] \\
& +\frac{1}{4}\left[p_{41} u_{1}^{3}+\left(p_{62}-p_{72}-p_{42}\right) v_{1}^{3}+\left(p_{62}+p_{72}-p_{42}\right) u_{1}^{2} v_{1}+\left(p_{41}+2 p_{71}\right) u_{1} v_{1}^{2}\right] \\
\dot{v}_{1}= & u_{1}\left[p_{1}-p_{5}+\sigma+\frac{1}{4}\left(p_{22}+p_{32}-p_{82}\right) a_{2}^{2}\right] \\
& +v_{1}\left[-\frac{1}{2}\left(c_{a 2}+2 \xi_{2} \omega_{2}\right)+\frac{1}{4}\left(p_{31}-p_{21}\right) a_{2}^{2}\right] \\
& +\frac{1}{4}\left[\left(p_{42}-p_{62}-p_{72}\right) u_{1}^{3}+p_{41} v_{1}^{3}+\left(p_{41}-2 p_{71}\right) u_{1}^{2} v_{1}+\left(p_{42}-p_{62}+p_{72}\right) u_{1} v_{1}^{2}\right] \\
\dot{a}_{2}= & \frac{1}{4} a_{2}\left[-2\left(c_{a 2}+2 \xi_{2} \omega_{2}\right)+p_{81} a_{2}^{2}+\left(p_{61}+p_{71}\right) u_{1}^{2}+\left(p_{61}-p_{71}\right) v_{1}^{2}-2 p_{72} u_{1} v_{1}\right]
\end{aligned}
$$

On the contrary, by letting $A_{1}=\frac{1}{2} a_{1} \exp \left(\mathrm{i} v_{1}\right)$ and $A_{2}=\frac{1}{2}\left(u_{2}+\mathrm{i} v_{2}\right) \exp \left(\mathrm{i} v_{2}\right)$, with $v_{1}=\alpha_{1}$ and $v_{2}=\alpha_{2}+\sigma t$, and performing some algebraic manipulations, a second standard form set of equations in the $\mathbf{x}:=\left(a_{1}, u_{2}, v_{2}\right)$ variables, suitable for the study of the stability of solutions with $a_{2}=0$, is obtained:

$$
\begin{aligned}
\dot{a}_{1}= & \frac{1}{4} a_{1}\left[-2\left(c_{a 1}+2 \xi_{1} \omega_{1}\right)+p_{41} a_{1}^{2}+\left(p_{21}+p_{31}\right) u_{2}^{2}+\left(-p_{21}+p_{31}\right) v_{2}^{2}-2 p_{22} u_{2} v_{2}\right] \\
\dot{u}_{2}= & u_{2}\left[-\frac{1}{2}\left(c_{a 2}+2 \xi_{2} \omega_{2}\right)+\frac{1}{4}\left(p_{61}+p_{71}\right) a_{1}^{2}\right] \\
& +v_{2}\left[p_{1}-p_{5}+\sigma+\frac{1}{4}\left(-p_{62}+p_{72}+p_{42}\right) a_{1}^{2}\right] \\
& +\frac{1}{4}\left[p_{81} u_{2}^{3}+\left(p_{82}-p_{22}+p_{32}\right) v_{3}^{3}+\left(p_{22}+p_{32}-p_{82}\right) u_{2}^{2} v_{2}+\left(p_{81}+2 p_{21}\right) u_{2} v_{2}^{2}\right] \\
\dot{v}_{2}= & u_{2}\left[p_{5}-p_{1}-\sigma+\frac{1}{4}\left(p_{62}+p_{72}-p_{42}\right) a_{1}^{2}\right] \\
& +v_{2}\left[-\frac{1}{2}\left(c_{a 2}+2 \xi_{2} \omega_{2}\right)+\frac{1}{4}\left(p_{61}-p_{71}\right) a_{1}^{2}\right] \\
& +\frac{1}{4}\left[\left(p_{82}-p_{22}-p_{32}\right) u_{2}^{3}+p_{81} v_{2}^{3}+\left(p_{81}-2 p_{21}\right) u_{2}^{2} v_{2}+\left(p_{82}+p_{22}-p_{32}\right) u_{2} v_{2}^{2}\right]
\end{aligned}
$$

The real amplitude-phase modulation equations (11), (12) or (13) can be analytically or numerically studied to find branches of fixed points (corresponding to limit cycles for the original system) and branches of periodic solutions (corresponding to quasi-periodic motions for the original system).

\section{A sample case study}

An extended numerical analysis has been performed on a real sample cable, already analyzed in the linear range [3], having axial stiffness $E A=29.7 \times 10^{6} \mathrm{~N}$, torsional stiffness $G J=159 \mathrm{Nm}^{2}$, bending stiffness $E I=2100 \mathrm{Nm}^{2}$, diameter $D=0.0281 \mathrm{~m}$, length $\ell \simeq 267 \mathrm{~m}$, sag $d=6.18 \mathrm{~m}$ and mass per unit length (including ice accretion) $m=1.80 \mathrm{~kg} / \mathrm{m}$.

The cable is close to the first cross-over point; its first natural frequencies under no-wind conditions are: $\omega_{s i}=2.809 \mathrm{rad} / \mathrm{s}, \omega_{a i}=2.799 \mathrm{rad} / \mathrm{s}, \omega_{s o}=1.399 \mathrm{rad} / \mathrm{s}$ and $\omega_{a o}=2.799 \mathrm{rad} / \mathrm{s} \quad(s=\mathrm{symmetric}, \quad a=$ anti-symmetric, $i=$ in-plane, $o=$ out-of-plane). Therefore, the first symmetric in-plane mode is involved in a 1:1 internal resonance with the first anti-symmetric in-plane and out-of-plane modes, and in a 2:1 internal resonance with the first symmetric out-of-plane mode. The associated eigenfunctions are plotted in Fig. 3. When the static component of the aerodynamic excitation acts on the cable, only the first symmetric in-plane frequency and relevant eigenfunction change. These alterations, which have been accounted for in the numerical analyses are, however, negligible for the case study. As a matter of fact, $\omega_{s i}$ varies in the range $(2.79,2.81) \mathrm{rad} / \mathrm{s}$ when $U$ spans the interval $(0,20) \mathrm{m} / \mathrm{s}$.

Assuming a Rayleigh structural damping, the proportionality factors $\alpha, \beta$ are arranged so that the first four cable modes have damping ratio coefficients equal to $\xi=0.44 \%$ [14], namely:

$$
\begin{aligned}
& \alpha=2 \xi \frac{\omega_{a i} \omega_{s o}}{\omega_{a i}+\omega_{s o}}=0.0082 \mathrm{~s} \\
& \beta=2 \xi \frac{1}{\omega_{a i}+\omega_{s o}}=0.0021 \mathrm{~s}
\end{aligned}
$$


(a)

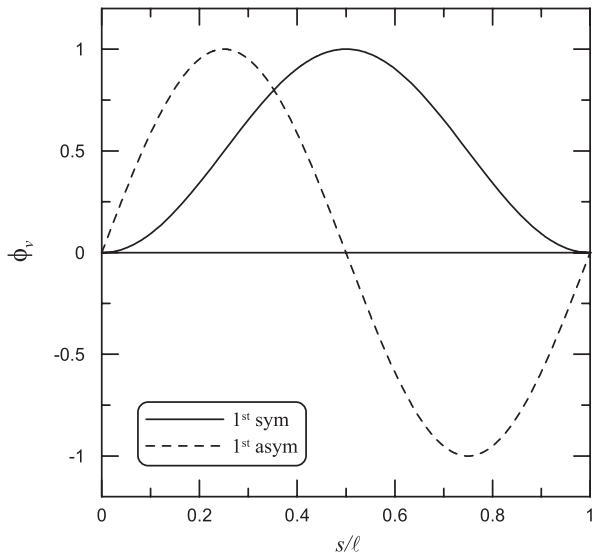

(b)

(c)
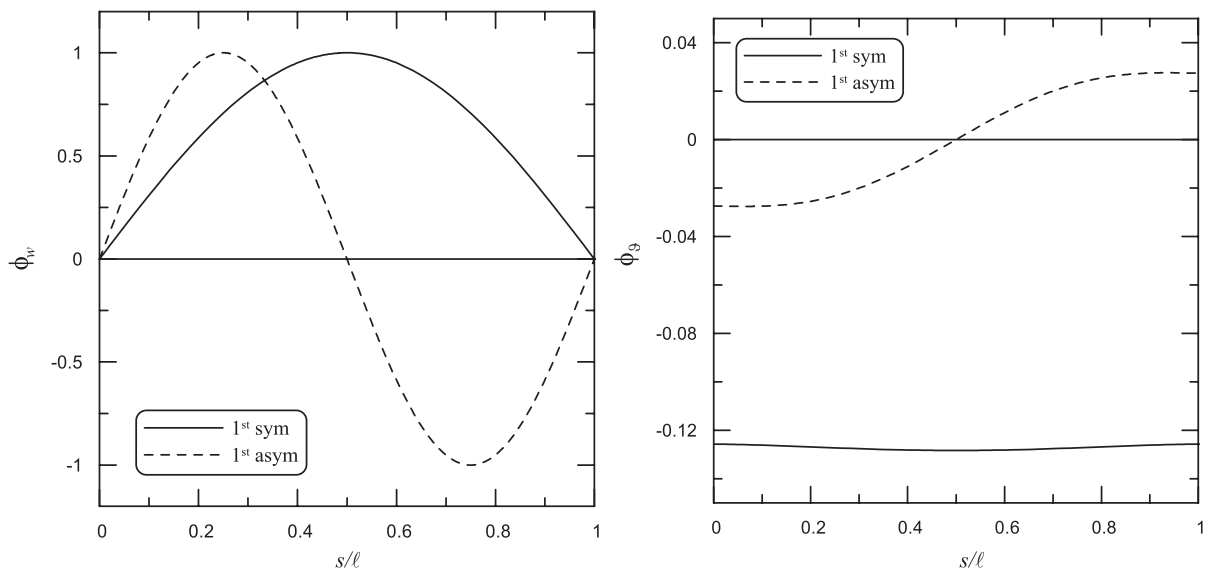

Fig. 3. Eigenfunctions of the Hamiltonian system: (a) in plane modes; (b) $w$ component and (c) $\vartheta$ component of the out of plane modes.

(a)

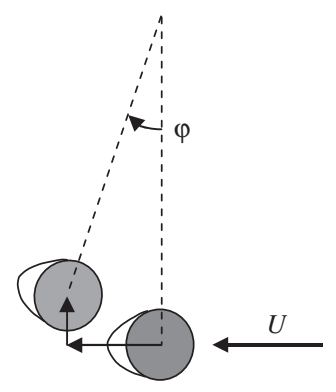

(b)

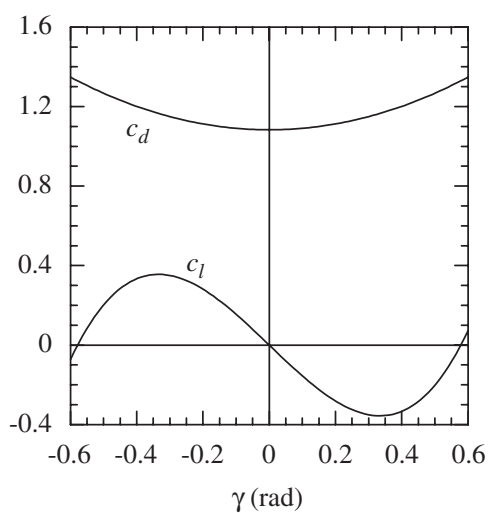

Fig. 4. U shaped conductor: (a) attitude of the cross section to wind ( $\varphi$ positive anticlockwise); (b) drag and lift aerodynamic coefficients.

With regard to aerodynamic properties, a U-shaped conductor having its maximum ice eccentricity opposite to the mean wind is considered (Fig. 4a, [14]). Its idealized aerodynamic coefficients are the drag coefficient $c_{d}(\gamma)=1.08334+0.735935 \gamma^{2}, \gamma$ being the angle of attack, and the lift $c_{l}(\gamma)=-1.5979 \gamma+4.77362 \gamma^{3}$ coefficient, that are assumed to be valid in the range $-0.6<\gamma<0.6 \mathrm{rad}$ (Fig. 4b); it is easy to verify that the 
cross-section is more prone to the galloping when the angle $\gamma$ is zero. Therefore, in order to exalt the nonlinear effects, the cross-section is rotated by $\gamma=-5^{\circ}=-0.0873 \mathrm{rad}$ under no-wind conditions; in this way it arrives, after the static swing, to the most dangerous attitude just close to the first galloping critical velocity [5].

\subsection{Critical conditions}

The conditions of incipient instability are investigated via an eigenvalue analysis carried out on the linear part of the discrete system (4). The eigenvalues are dependent on the mean wind velocity $U$ that governs the magnitude of the aerodynamic forces, included in matrices $\mathbf{C}$ and $\mathbf{H}$. When $U \rightarrow 0$ the eigenvalues tend to the Hamiltonian ones, modified by the structural damping, and therefore they have negative real parts. When $U$ increases and reaches a critical value $U_{c}$, one couple of complex conjugate eigenvalues crosses the imaginary axis and galloping oscillations occur (Hopf bifurcation).

Fig. 5 summarizes the results of this analysis, performed by a Galerkin model accounting for the first four in-plane and four out-of-plane modes $(m=4, n=4)$. It is seen that only the first two in-plane modes (the first symmetric and the first anti-symmetric) suffer instability, while the remaining ones are always stable. Critical conditions thus obtained have been validated using a finite-difference analysis by a Matlab ${ }^{\circledR}$ algorithm [3]; the solutions carried out by the finite-difference method are practically coincident with those obtained with a small number of modes. However, the crucial role played by the structural damping modeling should be noticed, since the third (symmetric) in-plane mode is very close to the instability domain (Fig. 5). Therefore, a different type of modeling (for instance, damping ratio constant over all the considered modes) would modify the critical conditions. It is worth noticing that both the critical modes show a first bifurcation point (Zoom A in Fig. 5), in which the instability phenomenon is triggered, and a second bifurcation point (Zoom B in Fig. 5), where a re-stabilization of the dynamical system occurs (transient galloping). It has been checked that the reentry bifurcation is due to the fact that an inclined equilibrium position of the suspended cable is considered [15]. Actually, the static swing $\varphi$ causes a continuous alteration of aerodynamic forces, which render the equilibrium unstable only for particular ranges of wind velocity. This fact is a concomitant cause in the stability recovery of the cable.

Thus, the instability occurrence is very different from the traditional 1 dof galloping analyses, in which the contribution of the mean wind force is neglected and the aerodynamic coefficients are assumed to be constant with velocity, at values more favorable for the occurrence of galloping; in those cases the transient galloping does not occur.
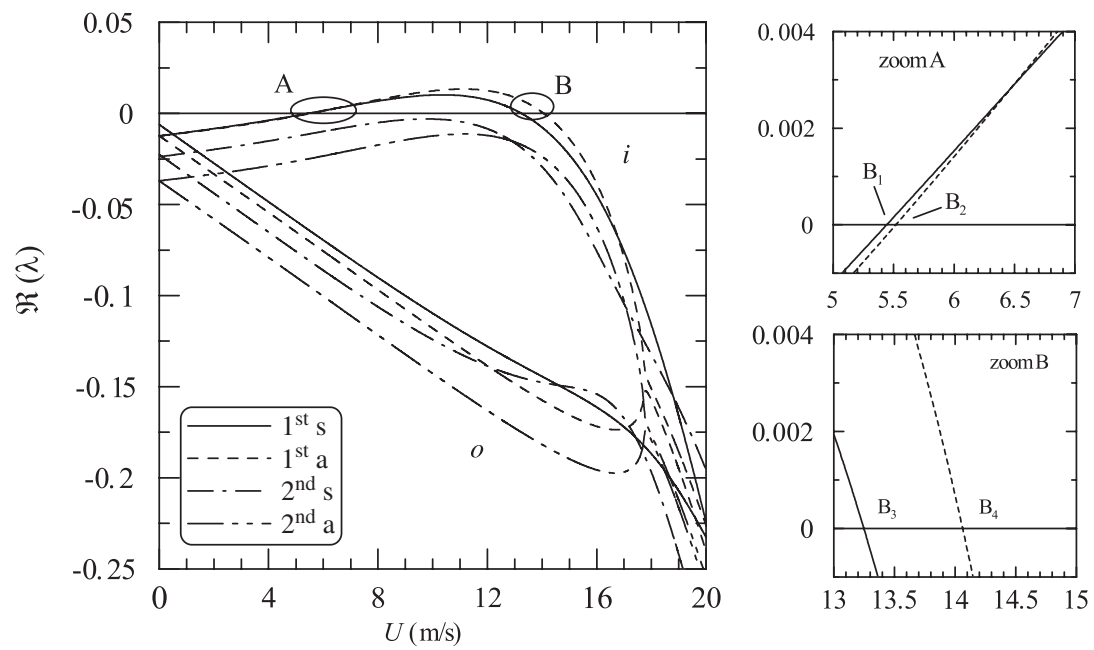

Fig. 5. Real parts of eigenvalues $\lambda$ of the linearized discrete system, taking into account the first eight modes of the cable ( $\mathrm{s}=$ symmetric, $\mathrm{a}=$ anti symmetric, $i=$ in plane, $o=$ out of plane). 


\subsection{Nonlinear behavior: numerical analysis}

The cable nonlinear behavior has been numerically analyzed. Attention has been focused on the instability domain in Fig. 5. A finite-difference analysis has first been performed, by discretizing the cable in $n=20$ nodes. The relevant stable steady-state solutions have been represented in Fig. 6a in terms of modal amplitudes, in order to compare the results with the Galerkin model. Amplitudes $a_{l}$ have been deduced by the maximum nodal displacements, after the transient is exhausted, according to the following criteria: the amplitude of the in-plane symmetric mode has been evaluated as $a_{1}:=\max _{t}[v(\ell / 2, t)]$, the amplitude of the inplane anti-symmetric mode as $a_{2}:=\max _{t}[(v(\ell / 4, t)-v(3 / 4 \ell, t)) / 2]$ and the amplitude of the out-of-plane antisymmetric mode as $a_{3}:=\max _{t}[(w(\ell / 4, t)-w(3 / 4 \ell, t)) / 2]$. Then the direct numerical integration of Galerkin discretized equations (4) has been carried out, either with a four degree-of-freedom model (first symmetric and anti-symmetric, in-plane and out-of-plane modes, $m=2$ and $n=2$, Fig. 6b) or with a two dof's model (the two first in-plane modes, $m=2$ and $n=0$, namely the only unstables, Fig. 6c).

The finite-difference model (Fig. 6a) and the four dof's Galerkin model (Fig. 6b) present very similar results from both qualitative and quantitative points of view. In both the cases, a branch of coupled solutions (indicated as II), consisting of anti-symmetric in-plane ( $a_{2}$, filled boxes) and out-of-plane ( $a_{3}$, empty boxes) components, is found; moreover, a branch of mono-modal symmetric in-plane solutions, indicated as IV- $a_{1}$ (filled circles), significant just for low wind velocities, takes place. Branches II and IV are coexistent and their

(a)

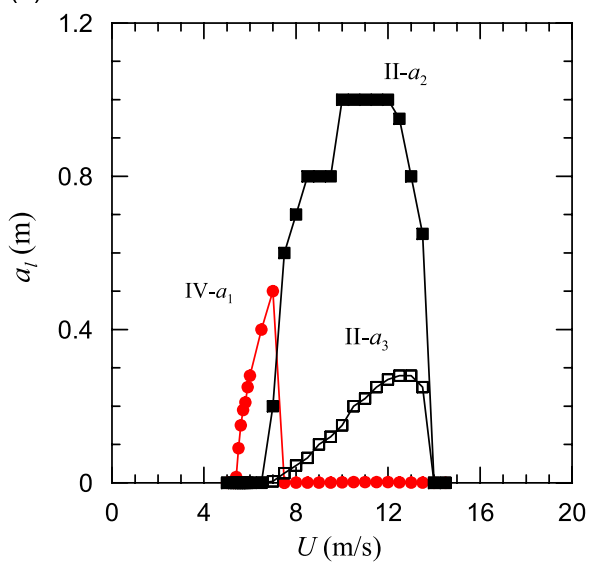

(b)

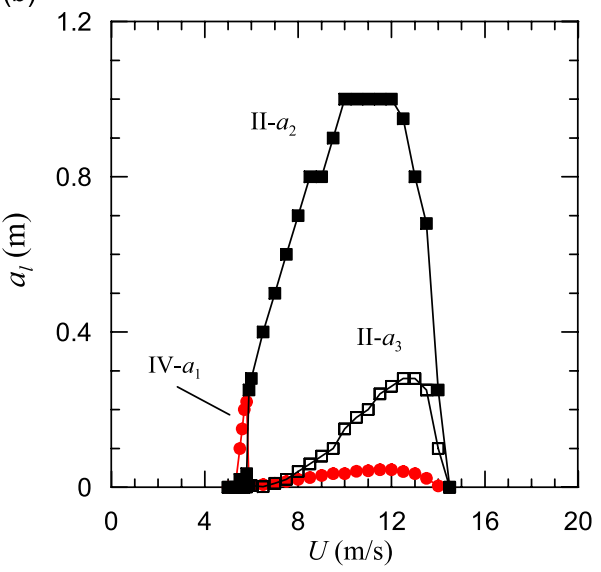

(c)

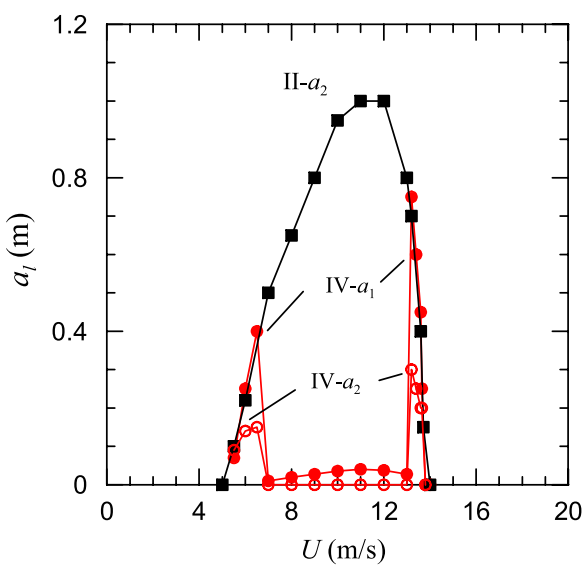

Fig. 6. Modal amplitudes drawn from: (a) the finite difference technique; (b) the direct integration of Galerkin 4 dof's model; (c) the direct integration of Galerkin 2 dof's model ( $a_{1}$ in plane symmetric amplitude, $a_{2}$ in plane anti symmetric amplitude, $a_{3}$ out of plane anti symmetric amplitude). 
occurrence is determined by the choice of the initial conditions. For a mean wind velocity greater than $\sim 6 \mathrm{~m} / \mathrm{s}$, the symmetric branch $\left(\mathrm{IV}-a_{1}\right)$ collapses to a very small amplitude, whereas the anti-symmetric one (II) persists and, for velocities greater than $\sim 8 \mathrm{~m} / \mathrm{s}$, the out-of-plane component $\left(a_{3}\right)$ significantly increases its amplitude. On the contrary, the contribution of the out-of-plane symmetric mode always seems negligible, which is triggered, as seen in Ref. [5], by the symmetric in-plane mode and probably suffers its premature breakdown.

In order to gain an insight into the interaction between the two most important modes, by insulating them from the remaining components, the simplest model with two sole in-plane dof has been studied. The amplitudes of the two modes are reported in Fig. 6c: branch II, now composed of the only in-plane component (filled boxes), is found; moreover, the coexisting branch IV also takes place describing, in this case, coupled inplane solutions of symmetric (IV- $a_{1}$, filled circles) and anti-symmetric (IV- $a_{2}$, empty circles) components. The branch IV shows significant amplitudes not only for low wind velocities $(U \in[5.5,7.0] \mathrm{m} / \mathrm{s})$ but also for high wind velocities $(U \in[13.0,14.0] \mathrm{m} / \mathrm{s})$. A certain scattering in the amplitude of the solutions pertaining to branch IV, not highlighted in the figure, is found and will be clarified in the next Section 4.3. If the existence of branch II and of the left part of branch IV suggests a good agreement between the three different models, the right part of the branch IV has been found only in the 2 dof's model. This is probably due to the fact that the basin of attraction of the right part of branch IV is very tiny. Therefore, it can be concluded that the reduced 2 dof's model seems able to reproduce the main behavior of the analyzed case study.

A clearer interpretation of this behavior is difficult to obtain by means of a purely numerical approach; however, it will be possible with the help of perturbation analysis (Section 4.3).

\subsection{Nonlinear behavior: perturbation analysis}

To better interpret the previous numerical analyses, the AME (11), (12) or (13), furnished by the MSM, relevant to the planar 2 dof's model, have been analyzed.

The fixed points $\left(a_{1}, a_{2}, \psi\right)$ of the dynamical system (11) have first been evaluated. The existence of the following branches is easily proved: branch I (trivial path): $a_{1}=a_{2}=0, \forall \psi$; branches II (mono-modal galloping), made of two sub-branches:

$$
\begin{array}{ll}
\mathrm{II}_{s}: & a_{1}=a_{1}(U)=\sqrt{\frac{2\left(c_{a 1}+2 \xi_{1} \omega_{1}\right)}{p_{41}}}, a_{2}=0, \forall \psi \\
\mathrm{II}_{a}: & a_{2}=a_{2}(U)=\sqrt{\frac{2\left(c_{a 2}+2 \xi_{2} \omega_{2}\right)}{p_{81}}}, a_{1}=0, \forall \psi
\end{array}
$$

Finally, branch III (bi-modal galloping): $a_{1}(U) \neq 0, a_{2}(U) \neq 0, \psi(U) \neq 0$. This latter branch is also found in an analytical way, as follows: the steady version of Eqs. (11) is transformed into a system of three linear algebraic equations in two unknowns $a_{1}^{2}$ and $a_{2}^{2}$, with coefficients depending on $\psi$; by requiring that the determinant of the matrix bordered by known terms vanishes, the two values of $\psi$ that lead to a bi-modal solution are drawn as functions of $U$. Fig. 7a shows the two branches $\mathrm{II}_{s}$ and $\mathrm{II}_{a}$, while Figs. 7b,c depict the two sub-branches III (compared with $\mathrm{II}_{a}$ and called III' $^{\prime}$ and III', $^{\prime \prime}$ respectively).

A stability analysis of fixed points has then been performed. The stability of branches III is governed by the eigenvalues of the Jacobian matrix of the polar form (11) of the RAME, evaluated at the equilibrium. Both the sub-branches III turn out to be unstable for the sample case study investigated here. The stability analysis of mono-modal branches $\mathrm{II}_{s}$ and $\mathrm{II}_{a}$ must be, in contrast, carried out on the mixed form (12) or (13) of the AME, according to the discussion carried out in Section 3.3.

When the stability of the branch $\mathrm{II}_{s}$ is studied, Eqs. (13) are linearized around $a_{1}=a_{1}(U)$ (Eq. (15) 1 ) and $u_{2}=v_{2}=0$. The variational equations thus obtained assume the form

$$
\left\{\begin{array}{l}
\delta \dot{a}_{1} \\
\delta \dot{u}_{2} \\
\delta \dot{v}_{2}
\end{array}\right\}=\left[\begin{array}{cc}
\lambda_{1} & 0 \\
0 & J_{a}
\end{array}\right]\left\{\begin{array}{l}
\delta a_{1} \\
\delta u_{2} \\
\delta v_{2}
\end{array}\right\}
$$


(a)

(b)

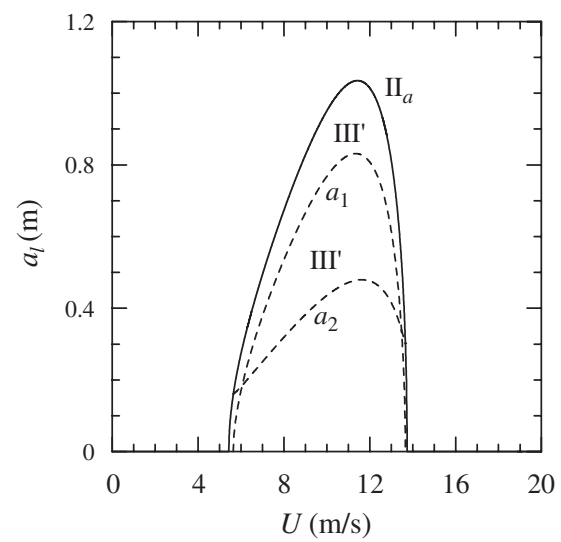

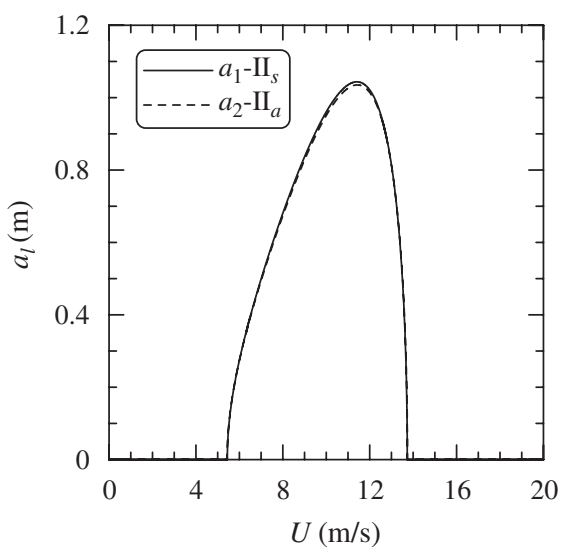

(c)

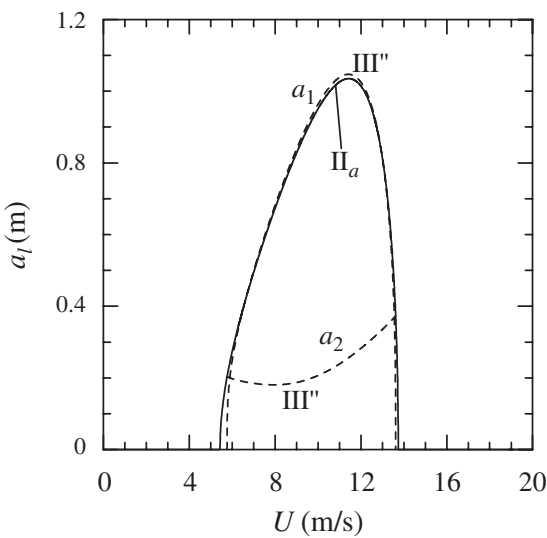

Fig. 7. Analytical modal amplitudes by MSM: (a) branches $\mathrm{II}_{s}$ and $\mathrm{II}_{a}$; (b) the sub branch $\mathrm{III}^{\prime}$ and (c) the sub branch $\mathrm{III}^{\prime \prime}$.

The eigenvalue $\lambda_{1}$ is always negative, meaning that the perturbation in the symmetric mode is decaying to zero. The eigenvalues $\lambda_{2}$ and $\lambda_{3}$ of the $2 \times 2$ block $J_{a}$, describing the evolution of the perturbation in the antisymmetric mode, must therefore be considered. For all the values of $U$ inside the range of existence of branch $\mathrm{II}_{s}$, namely $U \in[5.445,13.728] \mathrm{m} / \mathrm{s}, \lambda_{2}$ and $\lambda_{3}$ are complex-conjugate with a positive real part: it means that the equilibrium at this branch is everywhere unstable.

On the other hand, when the stability of branch $\mathrm{II}_{a}$ is investigated, system (12) is considered. The variational equations, based on $a_{2}=a_{2}(U)$ (Eq. (15) $)$ and $u_{1}=v_{1}=0$, appear in the form:

$$
\left\{\begin{array}{l}
\delta \dot{u}_{1} \\
\delta \dot{v}_{1} \\
\delta \dot{a}_{2}
\end{array}\right\}=\left[\begin{array}{cc}
J_{s} & 0 \\
0 & \lambda_{3}
\end{array}\right]\left\{\begin{array}{l}
\delta u_{1} \\
\delta v_{1} \\
\delta a_{2}
\end{array}\right\}
$$

The eigenvalue $\lambda_{3}$ is negative, meaning that the perturbation in the anti-symmetric mode is decaying. The eigenvalues $\lambda_{1}$ and $\lambda_{2}$ of the $2 \times 2$ block $J_{s}$ are therefore meaningful. It is found that, inside the region of existence of the branch $\mathrm{II}_{a}$, namely $U \in[5.434,13.738] \mathrm{m} / \mathrm{s}$, these eigenvalues are complex-conjugate with a negative real part, except for two small regions, $U \in[5.644,5.761] \mathrm{m} / \mathrm{s}$ and $U \in[13.617,13.661] \mathrm{m} / \mathrm{s}$, where they become real and distinct, and one of them assumes a positive value (see Fig. 8). The qualitative paths of the eigenvalues for increasing $U$ are also sketched in Figs. 8c and 8d. Therefore, two couples of bifurcation points are found, $\left(B_{1}^{\prime}, B_{2}^{\prime}\right)$ and $\left(B_{3}^{\prime}, B_{4}^{\prime}\right)$, that give rise to two small unstable regions on branch $\mathrm{II}_{a}$ (Fig. 9). 
(a)
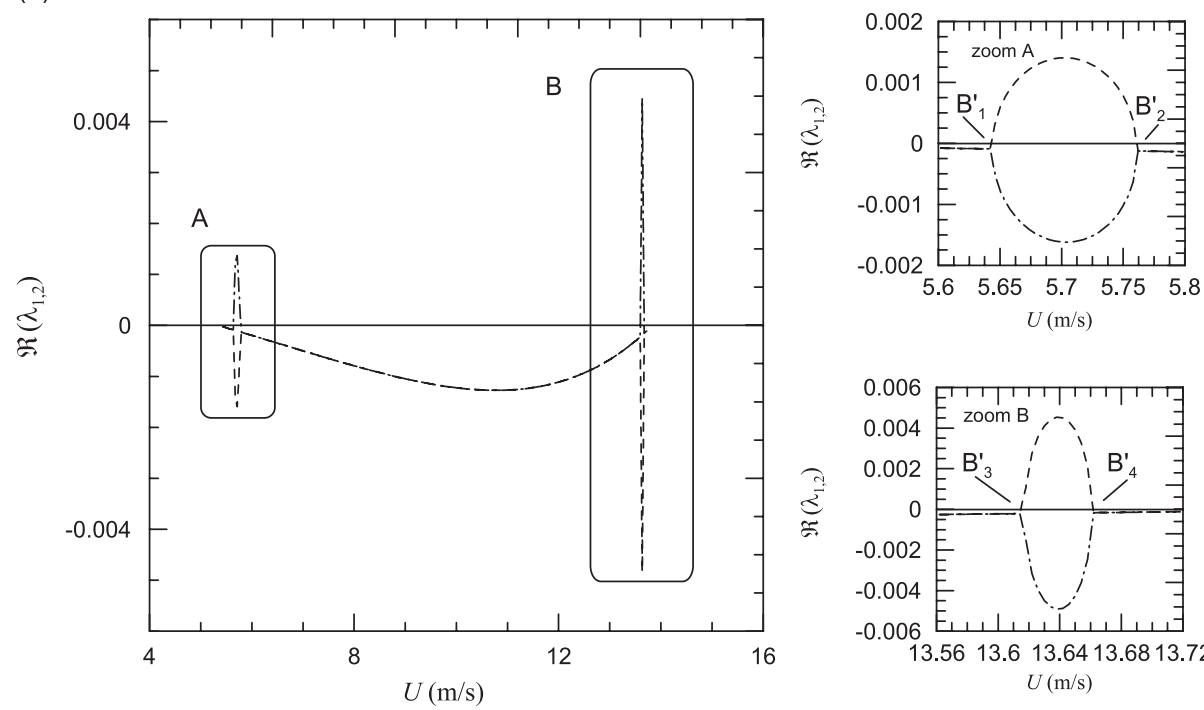

(b)
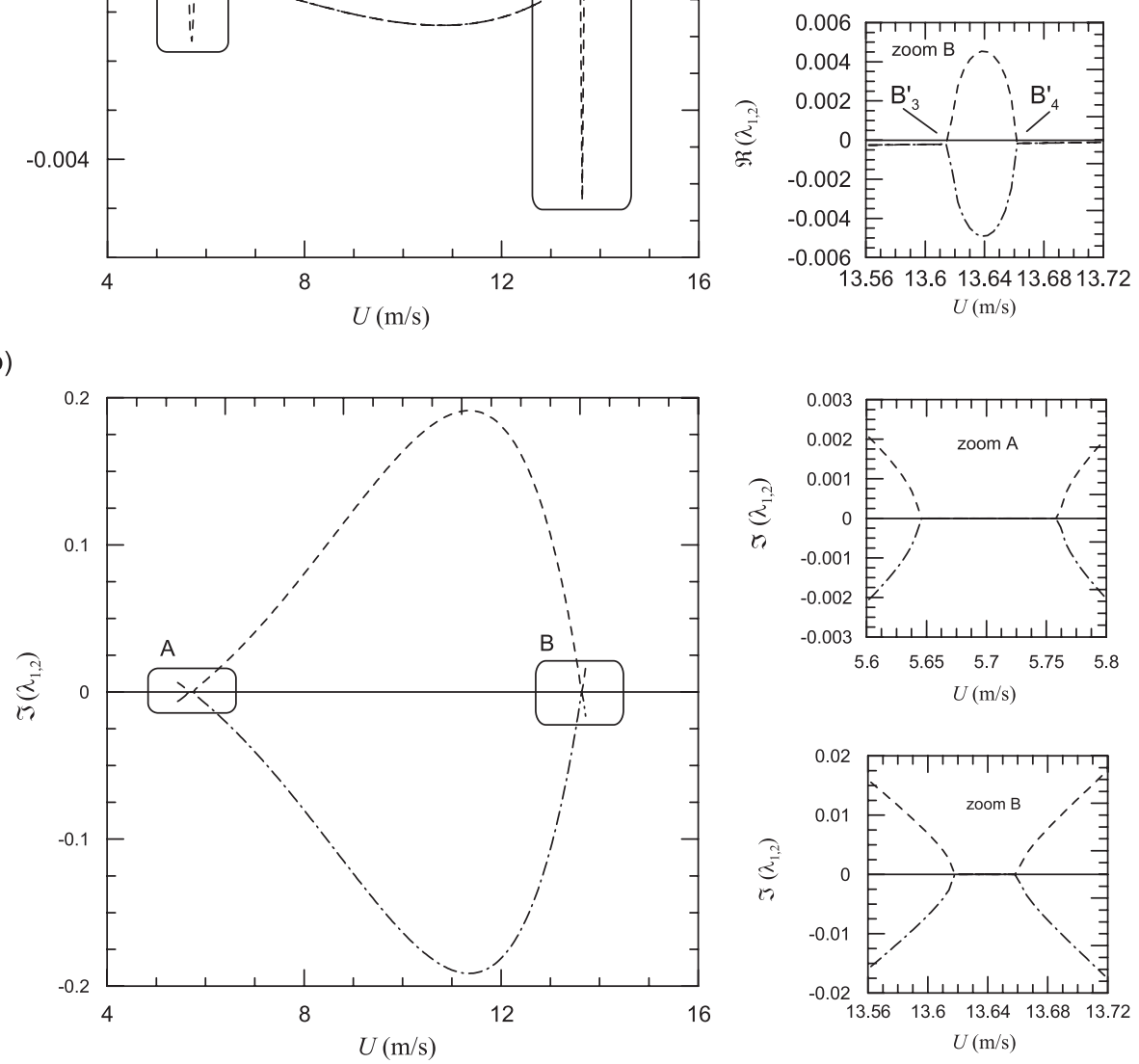

(c)
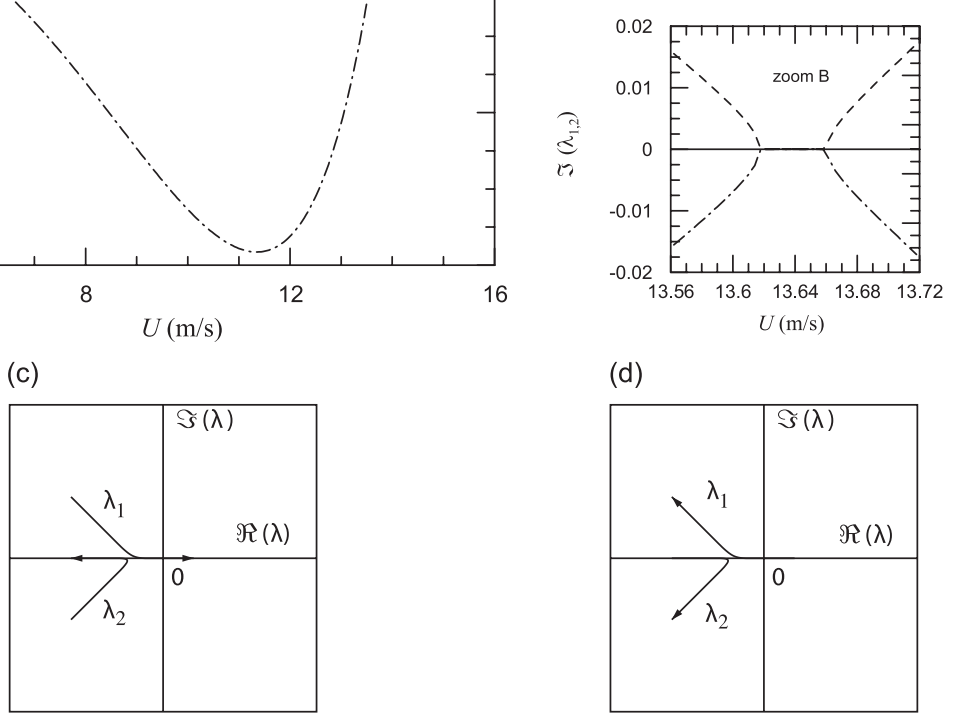

(d)

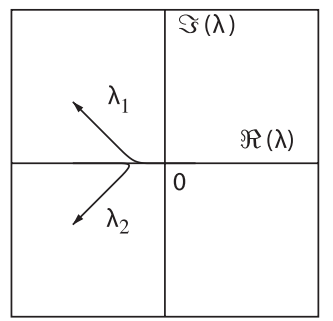

Fig. 8. Eigenvalues $\lambda_{1}$ and $\lambda_{2}$ for the study of the stability of the branch $\mathrm{II}_{a}$ : (a) real part, (b) imaginary part vs wind velocity (dashed line: $\lambda_{1}$, dashed dotted line: $\lambda_{2}$ ); qualitative paths on the complex plane for increasing $U$ : (c) near $B_{1}^{\prime}$ and $B_{3}^{\prime}$, (d) near $B_{2}^{\prime}$ and $B_{4}^{\prime}$.

These results are consistent with those obtained by numerical integrations of the RAME (11), after the transient is exhausted: (a) one never finds a steady solution consisting of $a_{1}$ different from zero and $a_{2}$ equal to zero, meaning instability of the branch $\mathrm{II}_{s}$; (b) out of the regions bounded by $B_{1}^{\prime}-B_{2}^{\prime}$ or by $B_{3}^{\prime}-B_{4}^{\prime}$, one finds 

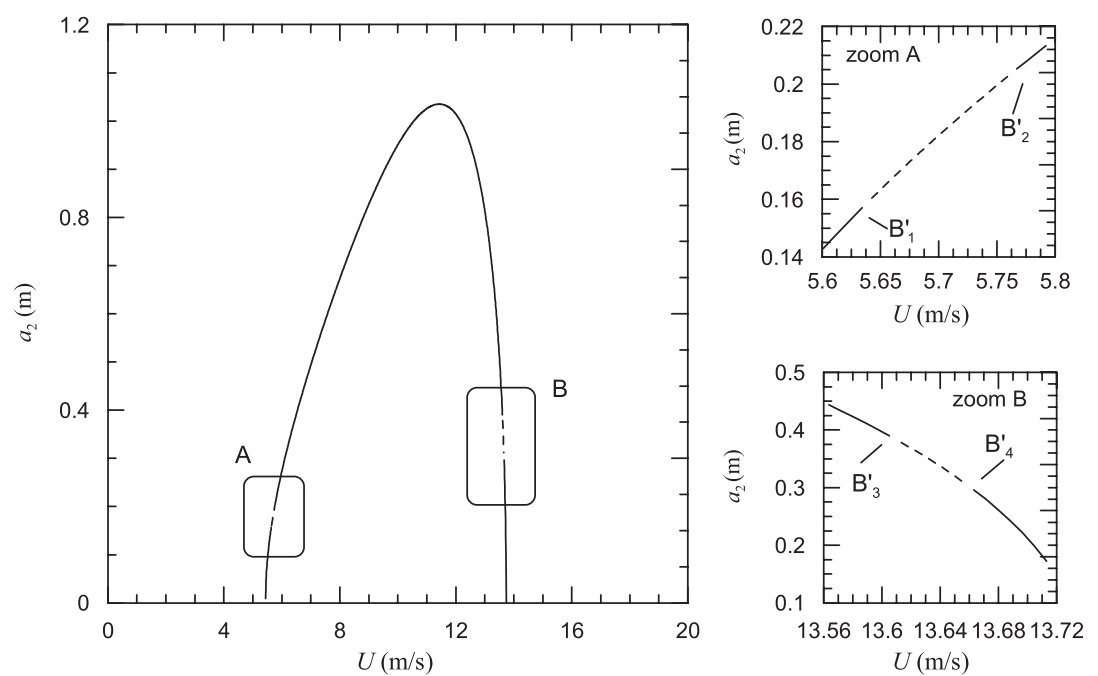

Fig. 9. Stability domains of the analytical branch $\mathrm{II}_{a}$ (continuous line: stable; dashed line: unstable).

(a)

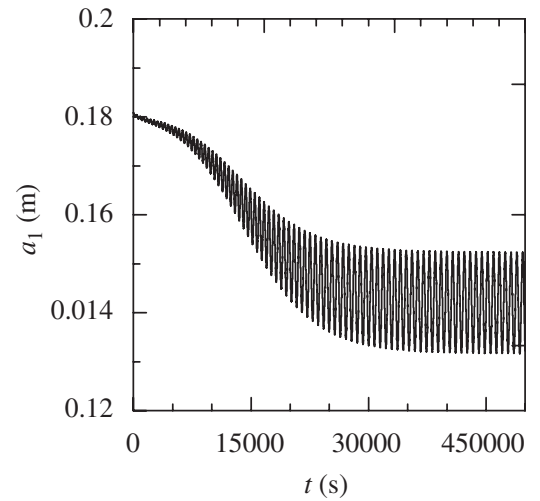

(b)

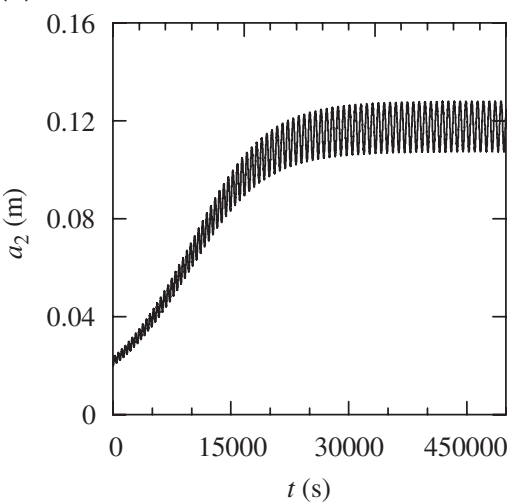

(c)

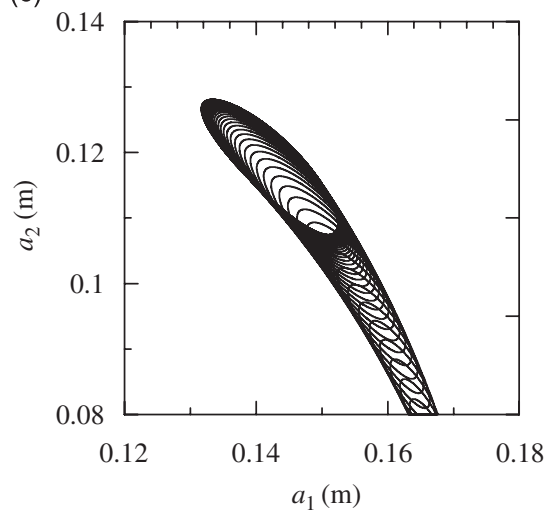

Fig. 10. Integration of the RAME (11) for $U=5.7 \mathrm{~m} / \mathrm{s}$, starting from $\mathrm{II}_{s}$ with perturbation equal to $0.02 \mathrm{~m}$ on $a_{2}$ and $0.02 \mathrm{rad}$ on $\psi$ : (a), (b) time histories; (c) trajectory $(t \in[11900,50000] \mathrm{s})$.

steady solutions consisting of $a_{2}$ different from zero and $a_{1}$ equal to zero, meaning stability of branch $\mathrm{II}_{a}$ in these zones; (c) inside the regions bounded by $B_{1}^{\prime}-B_{2}^{\prime}$ or by $B_{3}^{\prime}-B_{4}^{\prime}$, one finds only oscillating solutions, with both $a_{1}$ and $a_{2}$ different from zero, generating a limit cycle (Fig. 10): this means instability of branch II $_{a}$ in these zones; (d) in the close neighbors of the bifurcation points, the oscillating solutions with both $a_{1}$ and $a_{2}$ different from zero are also present in the stable range, coexisting with the branch $\mathrm{II}_{a}$ solution: the occurrence of oscillating or steady solutions depends on the initial conditions.

In order to show the effects of the amplitude modulations inside the zone $B_{1}^{\prime}-B_{2}^{\prime}$, the in-plane deflection of the cable $v(s, t)=a_{1}(t) \phi_{v_{1}}(s) \cos \left(\omega_{1} t\right)+a_{2}(t) \phi_{v_{2}}(s) \cos \left(\omega_{2} t+\alpha_{2}\right)$ is shown in Fig. 11 for different times $t \in[\underline{t}, \underline{t}+T], T$ being the slow-period. Only the solution $\alpha_{2}=0$ (in-phase motion) is displayed, since the other $\alpha_{2}=\pi$ solution (opposite-in phase motion) leads to mirror deflections around the midspan. The quasi-periodic nature of this class of motion (note the motion of the node) and the exchange of energy among the two contributing modes are evident.

To better understand the behavior of the system near the zones $B_{1}^{\prime}-B_{2}^{\prime}$ and $B_{3}^{\prime}-B_{4}^{\prime}$, the software package AUTO [16] has been used to analyze the AME (12). The results are presented in Fig. 12a,b, the first describing what happens near the zone $B_{1}^{\prime}-B_{2}^{\prime}$ and the second near the zone $B_{3}^{\prime}-B_{4}^{\prime}$. In these figures, the branch $\mathrm{II}_{a}$ is reported as a continuous line, the sub-branch III' (Fig. 7b) is reported as a dashed line and the sub-branch III" 


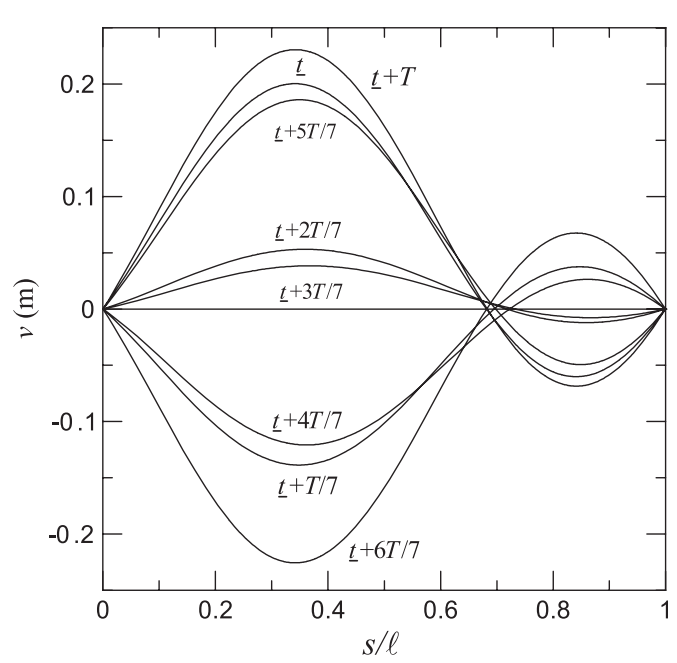

Fig. 11. Displacement $v(s, t)$ for $U=5.76 \mathrm{~m} / \mathrm{s} ; \underline{t}=59,300 \mathrm{~s} ; T=600 \mathrm{~s}$ (slow period) and $\alpha_{2}=0$.

(a)

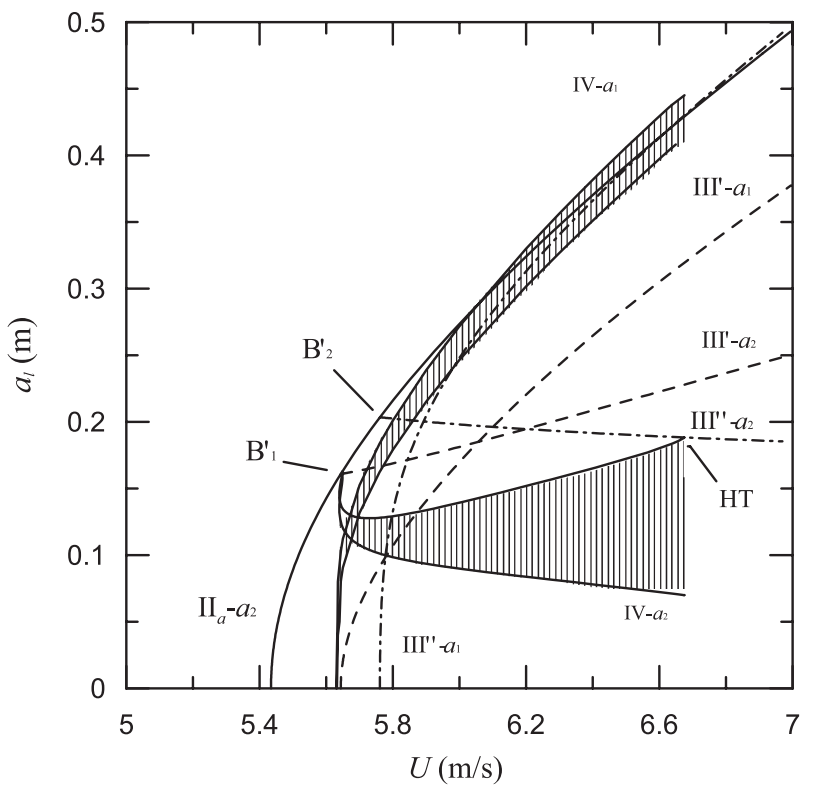

(b)

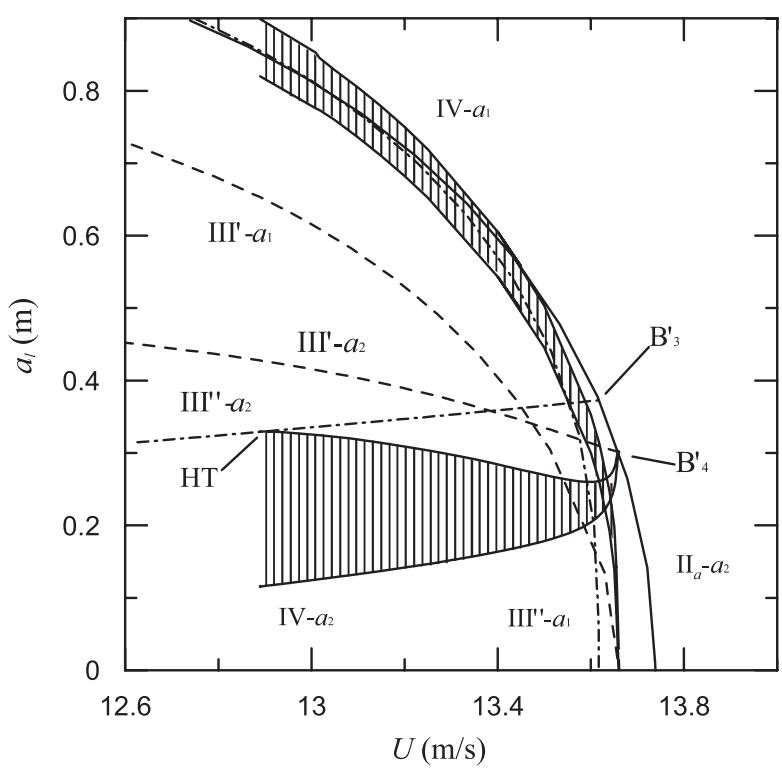

Fig. 12. Details of the MSM modal amplitudes as obtained by AUTO: (a) near the first instability zone ; (b) near the second instability zone.

(Fig. 7c) is reported as a dashed-dotted line, both distinguishing between the $a_{1}$ and $a_{2}$ components. A further branch of coupled periodic motions in the amplitudes, which is always stable in its domain, is found. It is called IV and reported as a hatched region bordered by continuous tiny lines; the hatching in the diagrams points out the difference between the maximum and the minimum amplitude in the $a_{1}$ and $a_{2}$ components on branch IV. It can be noticed that the external bifurcation points $\left(B_{1}^{\prime}\right.$ and $\left.B_{4}^{\prime}\right)$ are the intersections between branch $\mathrm{II}_{a}$ and the $a_{2}$ component of the unstable branch III', while the internal bifurcation points $\left(B_{2}^{\prime}\right.$ and $\left.B_{3}^{\prime}\right)$ are the intersections between branch $\mathrm{II}_{a}$ and the $a_{2}$ component of the unstable branch III". On the other hand, the bi-modal branch IV arises from the external bifurcation points, retaining its stability for the whole windvelocity range between the values of $B_{1}^{\prime}$ and $B_{2}^{\prime}$ and the values of $B_{3}^{\prime}$ and $B_{4}^{\prime}$. The bi-modal branch IV disappears when the $a_{2}$ periodic component touches the $a_{2}$ component of the unstable branch III", in a homoclinic tangency (indicated as HT). 
(a)

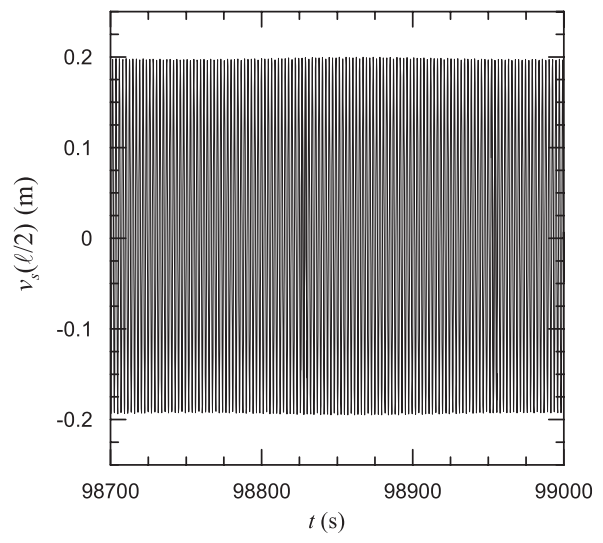

(b)

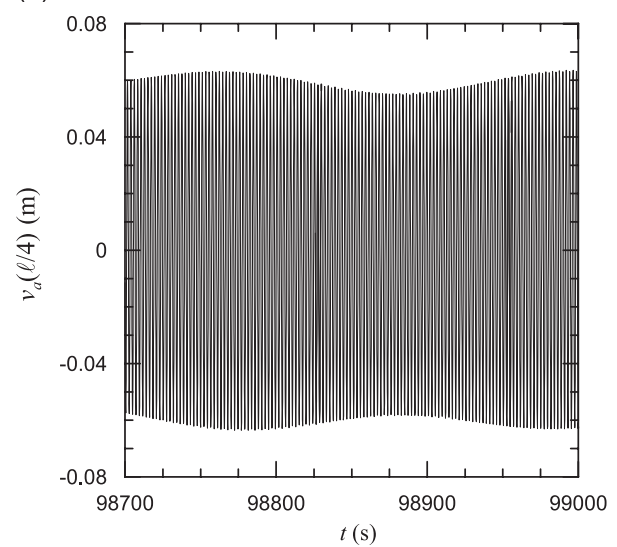

Fig. 13. Numerical integration of the finite difference model for $U=5.76 \mathrm{~m} / \mathrm{s}$ : (a) symmetric in plane displacement in $s=\ell / 2$; (b) anti symmetric in plane displacement in $s=\ell / 4$.

The existence of unstable, small regions inside branch $\mathrm{II}_{a}$ is also in agreement with the results obtained by the finite-difference model and it is able to explain the apparent scattering of the numerical results for a mean wind velocity of around $6 \mathrm{~m} / \mathrm{s}$ (Section 4.2). For values of $U$ corresponding to the instability region of the branch $\mathrm{II}_{a}$, nonlinear oscillations with varying amplitude, namely the branch IV, are also found numerically (Fig. 13). It has been noticed that these solutions exist outside the instability regions too: one can be misled by the very small variation of the amplitude of the symmetric oscillation and by the small amplitude of the antisymmetric oscillation, resembling the occurrence of a fake symmetric stable branch in the range $U \in$ $[5.45, \sim 7.00] \mathrm{m} / \mathrm{s}$ (see Fig. 6). Similar results, not reported here for the sake of brevity, have also been found through numerical direct integration of the Galerkin discrete models.

\section{Conclusions and prospects}

In this paper the galloping instability of a sagged suspended cable has been tackled using a consistent model of a curved cable-beam, geometrically nonlinear and able to torque. The nonlinear behavior of the cable has been studied through three alternative approaches: two numerical, with different types of discretization, and one analytical, through a perturbation method. Comparisons on a sample case study point out the importance of an extensive analysis of critical conditions and of the internal resonance conditions, which can lead to interactions among the modes in the nonlinear field. In particular, the classic galloping mode of the technical literature (first symmetric in-plane mode) actually becomes unstable for the simultaneous unstabilization of the anti-symmetric planar mode, in resonance with it.

The three different methods of analysis appear complementary in understanding the results, and highlight a good concordance among them, from both qualitative and quantitative points of view. The analytical approach seems particularly important in order to recognize situations of difficult interpretation with the sole numerical tools (for instance, unstable regions in fixed-point branches, with slight variations in amplitude). The use of a multimodal approach (like the finite-difference technique) leads to small, quantitative differences with the Galerkin method if the active modes are properly chosen.

The prospects of this research are many. The development of analytical MSM solutions, taking into account a higher number (e.g. four) of degrees of freedom, can allow recovery of out-of-plane displacements for complete comparison with numerical multimodal solutions. The analysis of different aerodynamic coefficients (i.e. different cable cross-sections) can permit underlining the actual contribution of the dynamic angle of torsion that produces instead moderate changes in the present case study. Finally, the possibility of analyzing cables in a larger neighborhood of the first cross-over point is of particular interest, since it can allow evaluation of the influence of geometric parameters on the multimodal galloping conditions. 


\section{Acknowledgments}

This work has been partially supported by the Italian Ministry of University (MIUR) through a PRIN co-financed program (www.disg.uniromal.it/fendis) and by the INTAS Project no. 06-1000013-9019 (www.intas.be).

\section{Appendix A. Equilibrium configuration}

Let us consider the cable in the equilibrium reference configuration $\mathscr{C}$ (see Fig. 1a), in which it is loaded by its own weight $\mathbf{b}_{0}$ and by the steady-state part $\mathbf{b}_{a}(\varphi, U)$ of the aerodynamic force. Since $\mathscr{C}$ is planar (Section 2$)$, equilibrium requires that the resultant force $\mathbf{b}(\varphi, U):=\mathbf{b}_{a}(\varphi, U)+\mathbf{b}_{0}$ lies in the plane of the cable. By making the force component vanish along the binormal direction, i.e. enforcing $\mathbf{b}(\varphi, U) \cdot \mathbf{a}_{3}=0$, it follows that

$$
\sin \varphi=-\frac{\rho_{a} U^{2} r}{2 m g}\left[c_{l}(\gamma-\varphi) \sin \varphi+c_{d}(\gamma-\varphi) \cos \varphi\right]
$$

where $\rho_{a}$ is the air density, $r$ a characteristic dimension of the cable cross-section and $g$ the gravity acceleration. Eq. (18) implicitly defines the nonlinear, non-trivial equilibrium path $\varphi=\varphi(U)$. The corresponding axial stress in the reference configuration assumes the following expression:

$$
T(\varphi, U)=\frac{\ell^{2}}{8 d}\left[\frac{1}{2} \rho_{a} r U^{2}\left(c_{l}(\gamma-\varphi) \cos \varphi-c_{d}(\gamma-\varphi) \sin \varphi\right)+m g \cos \varphi\right]
$$

\section{Appendix B. Galerkin discretized equations $(m=2, n=0)$}

Thee discretized equations obtained via the Galerkin standard approach, taking into account two sole active in-plane modes $(m=2, n=0)$, assume the following expression:

$$
\begin{aligned}
& \ddot{q}_{1}+2 \xi_{1} \omega_{1} \dot{q}_{1}+\omega_{1}^{2} q_{1}+c_{a 1} \dot{q}_{1}+h_{1} q_{1}^{2}+h_{2} q_{2}^{2}+h_{3} q_{1} q_{2}^{2}+h_{4} q_{1}^{3} \\
& \quad+d_{1} \dot{q}_{1}^{2}+d_{2} \dot{q}_{2}^{2}+d_{3} \dot{q}_{1}^{3}+d_{4} \dot{q}_{1} \dot{q}_{2}^{2}=0 \\
& \ddot{q}_{2}+2 \xi_{2} \omega_{2} \dot{q}_{2}+\omega_{2}^{2} q_{2}+c_{a 2} \dot{q}_{2}+h_{5} q_{1} q_{2}+h_{6} q_{1}^{2} q_{2}+h_{7} q_{2}^{3} \\
& \quad+d_{5} \dot{q}_{1} \dot{q}_{2}+d_{6} \dot{q}_{1}^{2} \dot{q}_{2}+d_{7} \dot{q}_{2}^{3}=0
\end{aligned}
$$

where $q_{1}$ and $q_{2}$ are the in-plane displacement amplitudes of the first symmetric mode and the first antisymmetric mode, respectively; $\xi_{1}$ and $\xi_{2}$ are the structural damping ratios; $\omega_{1}$ and $\omega_{2}$ are the circular frequencies, depending on the cable stress via the mean wind velocity $U ; c_{a 1}$ and $c_{a 2}$ are the aerodynamic damping coefficients; $h_{i}(i=1, \ldots, 7)$ are the nonlinear mechanical terms; and $d_{i}(i=1, \ldots, 7)$ are the nonlinear aerodynamic terms. It should be noted that this reduced system is uncoupled on the linear part, so that the critical wind velocity $U_{c}$ is given by the classic Den Hartog's criterion (namely, $U_{c}$ makes the total damping equal to zero). Different from the classic case, however, the aerodynamic coefficients are not constant in the present treatment, but they vary as a function of the static swing $\varphi$, which in turn is a function of the mean wind velocity $U$. The coefficients in Eqs. (20) are expressed as

$$
\begin{aligned}
& c_{a 1}=-\frac{b_{2 \dot{v}}}{m}, \quad h_{1}=-\frac{E A \kappa}{2 \ell} \frac{I_{1} I_{4}}{m I_{2}}+\frac{E A \kappa}{\ell} \frac{I_{1} I_{3}}{m I_{2}}, \quad h_{2}=-\frac{E A \kappa}{2 \ell} \frac{I_{1} I_{5}}{m I_{2}} \\
& h_{3}=-\frac{E A}{2 \ell} \frac{I_{3} I_{5}}{m I_{2}}, \quad h_{4}=-\frac{E A}{2 \ell} \frac{I_{3} I_{4}}{m I_{2}}, \quad d_{1}=-b_{2 \dot{v}^{2}} \frac{I_{6}}{m I_{2}} \\
& d_{2}=-b_{2 \dot{v}^{2}} \frac{I_{7}}{m I_{2}}, \quad d_{3}=-b_{2 \dot{v}^{3}} \frac{I_{8}}{m I_{2}}, \quad d_{4}=-3 b_{2 \dot{v}^{3}} \frac{I_{9}}{m I_{2}}
\end{aligned}
$$




$$
\begin{aligned}
& c_{a 2}=-\frac{b_{2 \dot{v}}}{m}, \quad h_{5}=\frac{E A \kappa}{\ell} \frac{I_{1} I_{10}}{m I_{11}}, \quad h_{6}=-\frac{E A}{2 \ell} \frac{I_{4} I_{10}}{m I_{11}}, \\
& h_{7}=-\frac{E A}{2 \ell} \frac{I_{10} I_{12}}{m I_{11}}, \quad d_{5}=-2 b_{2 \dot{v}^{2}} \frac{I_{7}}{m I_{11}}, \quad d_{6}=-3 b_{2 \dot{v}^{3}} \frac{I_{9}}{m I_{11}}, \\
& d_{7}=-b_{2 \dot{v}^{3}} \frac{I_{13}}{m I_{11}}
\end{aligned}
$$

where the dynamic wind-force components are:

$$
\begin{aligned}
b_{2 \dot{v}} & =-\frac{1}{2} \rho_{a} r U\left(c_{d}-c_{l} \cos \varphi \sin \varphi+c_{d} \sin ^{2} \varphi-c_{d}^{\prime} \sin \varphi \cos \varphi+c_{l}^{\prime} \cos ^{2} \varphi\right) \\
b_{2 \dot{v}^{2}} & =-\frac{1}{16} \rho_{a} r\left[4 c_{l} \cos ^{3} \varphi-c_{d}(9 \sin \varphi+\sin 3 \varphi)+8 c_{d}^{\prime} \cos \varphi-4 c_{d}^{\prime \prime} \cos ^{2} \varphi \sin \varphi+4 c_{l}^{\prime \prime} \cos ^{3} \varphi\right] \\
b_{2 \dot{v}^{3}} & =-\frac{1}{12} \frac{\rho_{a} r}{U} \cos ^{3} \varphi\left[-\sin \varphi\left(c_{d}^{\prime}-3 c_{l}-3 c_{l}^{\prime \prime}+c_{d}^{\prime \prime \prime}\right)+\cos \varphi\left(c_{l}^{\prime}+3 c_{d}+3 c_{d}^{\prime \prime}+c_{l}^{\prime \prime \prime}\right)\right]
\end{aligned}
$$

$\rho_{a}$ being the air density, $r$ a characteristic dimension of the cable cross-section (typically the diameter) and $c_{d}$ and $c_{l}$ the drag and lift aerodynamic coefficients, respectively, which appear up to their third derivatives and are calculated with regard to the planar equilibrium configuration $\mathscr{C}$ (Fig. 1a), that is for an angle of attack $\gamma$ equal to $(-\varphi)$. Finally, the modal integrals are defined as follows:

$$
\begin{aligned}
& I_{1}=\int_{0}^{\ell} \phi_{v_{1}} \mathrm{~d} s, \quad I_{2}=\int_{0}^{\ell} \phi_{v_{1}}^{2} \mathrm{~d} s, \quad I_{3}=\int_{0}^{\ell} \phi_{v_{1}} \phi_{v_{1}}^{\prime \prime} \mathrm{d} s, \\
& I_{4}=\int_{0}^{\ell}\left(\phi_{v_{1}}^{\prime}\right)^{2} \mathrm{~d} s, \quad I_{5}=\int_{0}^{\ell}\left(\phi_{v_{2}}^{\prime}\right)^{2} \mathrm{~d} s, \quad I_{6}=\int_{0}^{\ell} \phi_{v_{1}}^{3} \mathrm{~d} s \\
& I_{7}=\int_{0}^{\ell} \phi_{v_{2}}^{2} \phi_{v_{1}} \mathrm{~d} s, \quad I_{8}=\int_{0}^{\ell} \phi_{v_{1}}^{4} \mathrm{~d} s, \quad I_{9}=\int_{0}^{\ell} \phi_{v_{1}}^{2} \phi_{v_{2}}^{2} \mathrm{~d} s, \\
& I_{10}=\int_{0}^{\ell} \phi_{v_{2}}^{\prime \prime} \phi_{v_{2}} \mathrm{~d} s, \quad I_{11}=\int_{0}^{\ell} \phi_{v_{2}}^{2} \mathrm{~d} s, \quad I_{12}=\int_{0}^{\ell}\left(\phi_{v_{2}}^{\prime}\right)^{2} \mathrm{~d} s, \\
& I_{13}=\int_{0}^{\ell} \phi_{v_{2}}^{4} \mathrm{~d} s
\end{aligned}
$$

where $\phi_{v_{1}}$ (dependent on $U$ ) and $\phi_{v_{2}}$ (independent of $U$ ) are the modal shapes [17].

\section{Appendix C. Coefficients of the amplitude equations}

The coefficients $p_{i}$ appearing in the amplitude equations (10) are:

$$
\begin{aligned}
& p_{1}=-\frac{\left(c_{a 1}+2 \xi_{1} \omega_{1}\right)^{2}}{8 \omega_{1}}, \quad p_{21}=-\frac{d_{4} \omega_{2}^{2}}{2} \\
& p_{22}=\frac{\Lambda_{2} h_{1}}{\omega_{1}}+\frac{\Lambda_{6} h_{2}}{\omega_{1}}+\frac{h_{3}}{2 \omega_{1}}+2 d_{1} \Lambda_{2} \omega_{2}+d_{2} \Lambda_{6} \omega_{2}-\frac{d_{2} \Lambda_{6} \omega_{2}^{2}}{\omega_{1}} \\
& p_{31}=-d_{4} \omega_{2}^{2} \\
& p_{32}=\frac{2 \Lambda_{4} h_{1}}{\omega_{1}}+\frac{h_{3}}{\omega_{1}}+d_{2} \omega_{2}\left(\Lambda_{5}-\Lambda_{6}\right)+\frac{d_{2} \omega_{2}^{2}+h_{2}}{\omega_{1}}\left(\Lambda_{5}+\Lambda_{6}\right) \\
& p_{41}=-\frac{3}{2} d_{3} \omega_{1}^{2}, \quad p_{42}=\frac{h_{1}}{\omega_{1}}\left(\Lambda_{1}+2 \Lambda_{3}\right)+\frac{3 h_{4}}{2 \omega_{1}}+2 d_{1} \Lambda_{1} \omega_{1} \\
& p_{5}=-\frac{\left(c_{a 2}+2 \xi_{2} \omega_{2}\right)}{8 \omega_{2}}, \quad p_{61}=-d_{6} \omega_{1}^{2}
\end{aligned}
$$




$$
\begin{aligned}
& p_{62}=\frac{d_{5} \omega_{1}}{2}\left(\Lambda_{5}-\Lambda_{6}\right)+\frac{\Lambda_{3} h_{5}}{\omega_{2}}+\frac{h_{6}}{\omega_{2}}+\frac{h_{5}+d_{5} \omega_{1}^{2}}{2 \omega_{2}}\left(\Lambda_{5}+\Lambda_{6}\right) \\
& p_{71}=-\frac{d_{6} \omega_{1}^{2}}{2} \\
& p_{72}=d_{5} \Lambda_{1} \omega_{1}+\frac{d_{5} \Lambda_{6} \omega_{1}}{2}+\frac{h_{5}}{2 \omega_{2}}\left(\Lambda_{1}+\Lambda_{6}\right)+\frac{h_{6}}{2 \omega_{2}}-\frac{d_{5} \Lambda_{6} \omega_{1}^{2}}{2 \omega_{2}} \\
& p_{81}=-\frac{3}{2} d_{7} \omega_{2}^{2}, \quad p_{82}=\frac{\Lambda_{2} h_{5}}{2 \omega_{2}}+\frac{\Lambda_{4} h_{5}}{\omega_{2}}+\frac{3 h_{7}}{2 \omega_{2}}+d_{5} \Lambda_{2} \omega_{2}
\end{aligned}
$$

where the $\Lambda_{i}$ coefficients assume the expressions:

$$
\begin{aligned}
& \Lambda_{1}=\frac{h_{1}-d_{1} \omega_{1}^{2}}{3 \omega_{1}^{2}}, \quad \Lambda_{2}=\frac{-h_{2}+d_{2} \omega_{2}^{2}}{\omega_{1}^{2}-4 \omega_{2}^{2}}, \quad \Lambda_{3}=-\frac{h_{1}+d_{1} \omega_{1}^{2}}{\omega_{1}^{2}} \\
& \Lambda_{4}=-\frac{h_{2}+d_{2} \omega_{2}^{2}}{\omega_{1}^{2}}, \quad \Lambda_{5}=-\frac{d_{5} \omega_{1} \omega_{2}-h_{5}}{\omega_{1}^{2}+2 \omega_{1} \omega_{2}}, \quad \Lambda_{6}=\frac{h_{5}+d_{5} \omega_{1} \omega_{2}}{\omega_{1}^{2}-2 \omega_{1} \omega_{2}}
\end{aligned}
$$

\section{References}

[1] R.D. Blevins, Flow-induced Vibration, second ed., Krieger Publishing Company, Florida, 2001.

[2] M. Novak, Aeroelastic galloping of prismatic bodies, Journal of Engineering Mechanics ASCE 95 (EM1) (1969) $115-141$.

[3] A. Luongo, D. Zulli, G. Piccardo, A linear curved-beam model for the analysis of galloping in suspended cables, Journal of Mechanics of Materials and Structures 2 (4) (2007) 675-694.

[4] A. Luongo, D. Zulli, G. Piccardo, A nonlinear model of curved beam for the analysis of galloping of suspended cables, in: B.H.V. Topping, G. Montero, R. Montenegro (Eds.), Proceedings of Eighth International Conference on Computational Structures Technology, Las Palmas de Gran Canaria, Spain, 2006.

[5] A. Luongo, D. Zulli, G. Piccardo, On the effect of twist angle on nonlinear galloping of suspended cables, Computers and Structures, (2008), doi:10.1016/j.compstruct.2008.04.014.

[6] C.L. Lee, N.C. Perkins, Nonlinear oscillations of suspended cables containing a two-to-one internal resonance, Nonlinear Dynamics 3 (1992) 465-490.

[7] C.L. Lee, J.C. Chao, Out-of-plane vibrations of curved non-uniform beams of constant radius, Journal of Sound and Vibration 238 (3) (2000) 443-458.

[8] G. Rega, W. Lacarbonara, A.H. Nayfeh, C.M. Chin, Multiple resonances in suspended cables: direct versus reduced-order models, International Journal of Non-Linear Mechanics 34 (1999) 901-924.

[9] S. Wolfram, The Mathematica Book, Wolfram Research Inc., 2003.

[10] Visual Numerics, IMSL Fortran Library User's Guide, 2003.

[11] R.W. Clough, J. Penzien, Dynamics of Structures, third ed., Computer \& Structures, Inc., Berkeley, CA, USA, 2003.

[12] A.H. Nayfeh, D.T. Mook, Nonlinear Oscillations, Wiley, New York, 1979.

[13] A. Luongo, A. Di Egidio, A. Paolone, On the proper form of the amplitude modulation equations for resonant systems, Nonlinear Dynamics 27 (2002) 237-254.

[14] P. Yu, Y.M. Desai, N. Popplewell, A.H. Shah, Three-degree-of-freedom model for galloping, part II: solutions, Journal of Engineering Mechanics, ASCE 119 (12) (1993) 2426-2448.

[15] A. Luongo, G. Piccardo, A continuous approach to the aeroelastic stability of suspended cables in 1:2 internal resonance, Journal of Vibration and Control 14 (1-2) (2008) 135-157.

[16] E.J. Doedel, AUTO-07P: Continuation and Bifurcation Software for Ordinary Differential Equation, 2007.

[17] H.M. Irvine, T.K. Caughey, The linear theory of free vibrations of a suspended cable, Proceedings of the Royal Society of London A 341 (1974) 299-315. 